\title{
DATOS PARA LA CONSERVACIÓN FLORÍSTICA EN FUNCIÓN DE LA AMPLITUD GEOGRÁFICA DE LAS ESPECIES EN EL SEMIDESIERTO QUERETANO, MÉXICO
}

\author{
Rafael Hernández-Magaña ${ }^{1}$, José Guadalupe \\ HERNÁNDEZ-ORIA ${ }^{2,4}$ Y RUTH CHÁVEZ ${ }^{3}$
}

${ }^{1}$ Plan de Ayutla 455, Colonia Adolfo López Mateos, 76750

Tequisquiapan, Querétaro, México.

${ }^{2}$ Ignacio Ramírez 12 Int. 41, Centro Histórico, 76150

Santiago de Querétaro, Querétaro, México.

${ }^{3}$ Lázaro Cárdenas 14, Centro, 76504 Cadereyta de Montes, Querétaro, México.

${ }^{4}$ Autor para la correspondencia: xerofilia@yahoo.com.mx

\section{RESUMEN}

Se presenta una lista de algunos elementos florísticos del Cuadrante Tolimán, una porción del Semidesierto Queretano en el extremo sur del Desierto Chihuahuense, México. Se registraron 219 especies y 11 subespecies, agrupadas en 155 géneros y 63 familias. Esta área relativamente pequeña alberga 51 especies endémicas, de las cuales 24 se encuentran en alguna categoría de riesgo de acuerdo con la NOM-059-SEMARNAT-2010. Con el propósito de contribuir a la protección de este contingente florístico, se determinaron las áreas prioritarias para la conservación a través de un análisis de complementariedad (diversidad beta), y mediante un índice que mide el área media de distribución se revela un patrón restringido en varios de los taxones. Este ensamblaje florístico prospera en un área con alta heterogeneidad ambiental, distribuido en ocho asociaciones vegetales identificadas a través de una clasificación numérica. Desafortunadamente la riqueza florística del Semidesierto Queretano no está legalmente protegida como reserva natural en la actualidad.

Palabras clave: áreas prioritarias, patrón de distribución, rareza, región semiárida.

\section{ABSTRACT}

A floristic list of 219 species and 11 subspecies, comprising 63 families and 155 genera occurring in the Toliman grid square; an area located within the Queretaroan Semi- 
arid region in the southern Chihuahuan Desert, Mexico, is provided. This relatively small area is characterized by a high endemicity of flowering plant species: the floristic sample harbors 51 endemic species, and 24 species are included in some kind of risk category according to the Mexican Norm NOM-059-SEMARNAT-2010. To contribute to the protection of this flora, we performed an iterative process of complementarity analysis (beta diversity) to determine sets of priority areas for its conservation. The ranges of all species were considered by using an index that measures the average range size to determine the degree of "restrictedness" of the species. The results show that the ranges of most of the species are restricted. This flora develops within an area of high environmental heterogeneity, grouped in eight plant associations recognized by means of a numerical classification and multivariate analysis of the communities occurring in the region. Unfortunately, at present the high richness and endemism of the Queretaroan Semi-arid region are not under the legal protection of any natural reserve.

Key words: distributional range, priority areas, rarity, semi-arid region.

\section{INTRODUCCIÓN}

Las regiones áridas y semiáridas de México contribuyen a la riqueza de la flora mexicana con cerca de 6000 especies de plantas vasculares, de las cuales alrededor de 60\% son endémicas (Rzedowski, 1978, 1991, 2005). Una de las comarcas secas más extensas en el territorio mexicano es el Desierto Chihuahuense, provincia biogeográfica reconocida como un reservorio biológico de notable importancia (Medellín, 1982) y una región prioritaria para la conservación al nivel mundial (Dinerstein et al., 1999). En la porción extrema sur y disyunta del cuerpo principal del Desierto Chihuahuense se localiza la Zona Árida Queretano-Hidalguense, cuyo segmento meridional corresponde al sector del Semidesierto Queretano (Sánchez et al., 2006), mismo que alberga al llamado “Cuadrante Tolimán” (Hernández y Bárcenas, 1995).

Los estudios florísticos circunscritos a esta región señalan que el área sustenta una considerable diversidad y un endemismo alto (Zamudio, 1984; Zamudio et al., 1992), así como gradientes ambientales que crean mosaicos variados de vegetación con un cúmulo importante de riqueza de especies (Fernández y Colmenero, 1997). A pesar de que podría considerarse como una zona relativamente bien inventariada, recientes exploraciones han añadido nuevos registros a los ya existentes, incrementando con ello la riqueza florística conocida en general, y en particular de la familia Cactaceae (Chávez et al., 2006; Hernández-Oria et al., 2007b). Así, se ha conforma- 
do una agrupación de plantas que integra diversos elementos con distintas afinidades geográficas y patrones de distribución particulares.

Un componente biogeográfico esencial de las especies es el tamaño de su área de distribución (Rapoport y Monjeau, 2001; Contreras-Medina, 2006), característica que usualmente ha sido utilizada para establecer criterios que definen estrategias de conservación biológica (Anderson, 1994). Para las zonas áridas y semiáridas de México se conocen algunos ejemplos en los cuales el tamaño del área de distribución ha sido un factor usado para catalogar, en términos espaciales, a las especies amenazadas y/o vulnerables (Villaseñor y Elias, 1995; Villaseñor et al., 1998; Dávila et al., 2002; Méndez-Larios et al., 2004). Generalmente las áreas de distribución pequeñas tienen mayor peso específico como prioridades para la conservación (Villaseñor, 2003; Méndez-Larios et al., 2005). En este contexto, si bien se considera al Cuadrante Tolimán como un espacio florístico trascendente por su diversidad y endemismo, se desconoce la magnitud de tales elementos, sobre todo del recambio de especies (diversidad beta), así como del ámbito geográfico de algunos elementos florísticos presentes. Este aspecto podría sustentar su protección formal como una reserva natural que alberga un ensamble importante de especies vegetales.

En este trabajo se cuantificó el área de distribución de los elementos florísticos registrados en el Cuadrante Tolimán para determinar su grado de amplitud. Una vez conocido este parámetro, se exploraron sus posibles relaciones con la riqueza, el endemismo y el recambio de especies (diversidad beta), y mediante un análisis de complementariedad con respecto a todos los sitios muestreados en el Cuadrante Tolimán, se obtuvo un esquema óptimo y espacialmente explícito de zonas prioritarias para la conservación en esta región. Por último, se aplicó una técnica multivariada de clasificación (Twinspan) a las muestras de especies registradas para determinar grupos o asociaciones vegetales y su distribución en el Cuadrante Tolimán, y se exploró la influencia de las variables físicas en la distribución de esas muestras mediante un análisis indirecto de gradiente.

\section{ÁREA DE ESTUDIO}

El Cuadrante Tolimán es un área de aproximadamente $2139 \mathrm{~km}^{2}$ comprendida entre la latitud $20^{\circ} 30^{\prime}-21^{\circ} 00^{\prime}$ norte y la longitud $99^{\circ} 30^{\prime}-100^{\circ} 00^{\prime}$ oeste, y abarca una porción de la Zona Árida Queretano Hidalguense (Rzedowski, 1978), que es una región disgregada del Desierto Chihuahuense en su extremo sur (Fig. 1). A este territorio en el estado de Querétaro se le conoce como Semidesierto Queretano y se 


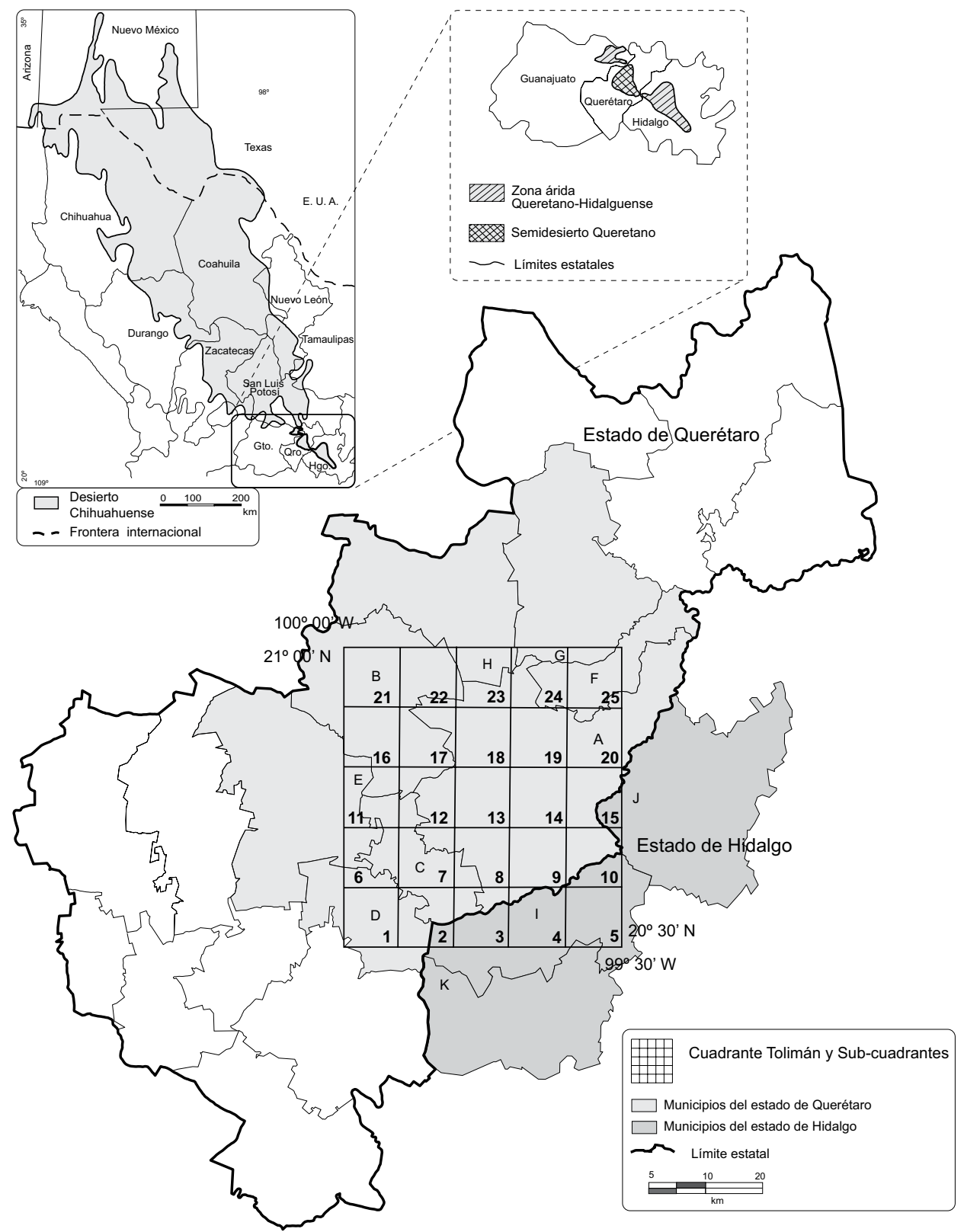

Fig. 1. Distribución del Desierto Chihuahuense, Semidesierto Queretano y ubicación del Cuadrante Tolimán. Municipios del estado de Querétaro: A. Cadereyta; B. Tolimán; C. Ezequiel Montes; D. Tequisquiapan; E. Colón; F. San Joaquín; G. Pinal de Amoles; H. Peñamiller. Municipios del estado de Hidalgo: I. Tecozautla; J. Zimapán; K. Huichapan. 
localiza en el área de confluencia de las provincias fisiográficas Sierra Madre Oriental, Mesa del Centro y Eje Neovolcánico, las cuales están asociadas a una litología sedimentaria, volcánica ácida y volcánica básica, respectivamente, así como a suelos de desarrollo pobre o moderado. La topografía regional está dominada por lomeríos de entre 1450 y $2100 \mathrm{~m}$ sobre el nivel del mar y el macizo montañoso de la sierra El Doctor, con elevaciones de entre 2250 y $3100 \mathrm{~m}$. El tipo de clima predominante es el semiseco templado con lluvias en verano ( $\mathrm{BS}_{1} \mathrm{kw}$ ), caracterizado por una temperatura media anual de entre 16 y $18{ }^{\circ} \mathrm{C}$, con una precipitación media anual de entre 400 y $600 \mathrm{~mm}$, mientras que en las partes elevadas el clima es templado subhúmedo $\left(\mathrm{Cw}_{\mathrm{o}}\right)$, con una precipitación anual de entre 600 y $1000 \mathrm{~mm}$ y una temperatura media anual de entre 10 y $12{ }^{\circ} \mathrm{C}$. (Anónimo, 1986). La vegetación regional presenta diversas variantes de matorral xerófilo (sensu Zamudio, 1984) y en las zonas templadas se encuentran los matorrales esclerófilo y submontano, al igual que bosques de Juniperus, Quercus y Pinus (Zamudio et al., 1992).

\section{MÉTODO}

Registro de especies

El muestreo se diseñó con base en el trabajo de Gómez-Hinostrosa y Hernández (2000), por lo que el área del Cuadrante Tolimán se dividió en 25 subcuadrantes de $6 \times 6$ minutos, equivalentes aproximadamente a $110 \mathrm{~km}^{2}$ cada uno (Fig. 1). Cada subcuadrante representa una unidad geográfica operativa (UGO, sensu Crovello, 1981) en su mínima dimensión espacial para el presente estudio y en cada uno de ellos se recorrieron tres transectos de al menos $3 \mathrm{~km}$ de longitud (en total 75 transectos), en los que se registró mediante observación a las especies presentes. Los sitios de muestreo se escogieron de acuerdo con los tipos de vegetación mejor representados en cada subcuadrante y con las áreas con mayor cubierta vegetal, según un mapa de uso de suelo (Anónimo, 2000). Tal tipo de protocolo, a través de transectos sobre una superficie preestablecida, no implica la colecta sistematizada de todas las plantas, sino que ésta se orientó hacia aquellas especies que no habían sido recolectadas con anterioridad, escasamente muestreadas o que no habían sido inventariadas previamente de la región. Con los datos de registros de las especies derivados de las observaciones de los transectos se construyó una matriz binaria (presencia/ausencia) de $n$ especies por subcuadrantes ( 25 en total) y se procedió a su análisis como contingente florístico. La nomenclatura utilizada para los tipos de vegetación fue la propuesta por Zamudio et al. (1992). 
Delimitación del conjunto florístico

El presente análisis contempla sólo a las plantas perennes que fueron recolectadas como se describe en el muestreo. Es una aproximación que pretende destacar algunos grupos relevantes de la flora del Semidesierto Queretano (e.g., cactáceas y leguminosas, entre otras), que además se distinguen por ser elementos más o menos raros, o con imprecisiones en sus áreas de distribución. Por consiguiente, el propósito no es documentar y analizar la lista acumulada de la flora regional que haya sido registrada a lo largo de los años en el Cuadrante Tolimán y áreas adyacentes (ver por ejemplo: Zamudio, 1984; Argüelles et al., 1991; Zamudio et al., 1992; Fernández y Colmenero, 1997).

Registro de variables ambientales

En cada uno de los tres transectos por subcuadrante se registraron las siguientes variables ambientales (Anónimo, 1986): latitud, longitud, altitud, geología (rocas ígneas extrusivas e intrusivas, sedimentarias), litología (volcanoclástico, basalto, toba, brecha volcánica, riolita, arenisca, caliza, caliza-lutita, arenisca-clonglomerado, granodiorita), suelo (litosol, rendzina, feozem, regosol, acrisol), porcentaje de pendiente, temperatura media anual, temperatura máxima y mínima, precipitación media anual, precipitación total y tipo de clima (seco-semicálido, semiseco-cálido, semiseco-semicálido, semiseco-templado, semicálido-subhúmedo y templado subhúmedo). Se exploró su valor explicativo con relación a la distribución de las muestras de vegetación en los subcuadrantes mediante un análisis canónico de correspondencias (ACC), una vez cotejada la respuesta unimodal de los datos mediante un análisis de correspondencia sin tendencia (ACT) (Jongman et al., 2000). Para este propósito se utilizó el programa MVSP 3.1 (Mutivariate Statistical Package, Kovach, 1999).

Índice de área promedio

Para evaluar la amplitud de distribución geográfica de las especies observadas en el Cuadrante Tolimán, se aplicó un índice que equivale al área promedio de distribución de una especie en un área dada (Santos y Arita, 2002), de acuerdo con la siguiente fórmula:

$$
I_{c}=\left(\sum_{i=1}^{n_{c}} 1 / \mathrm{A}\right) / n_{c}
$$

donde $I_{c}$ es el valor del índice para el área $c, A$ es el área de distribución de la especie $i$, y $n_{c}$ es el número de especies en la misma. El área promedio de distribución de 
una especie se determinó según el número de veces (entre 1 y 25) en las que estuvo presente en los subcuadrantes que integraron la matriz. Cada presencia tomó el valor del área $A$ (110, excluyendo unidades) que ocupa cada subcuadrante. Así, la magnitud total del área de distribución $(A)$ de una especie equivale a la sumatoria del valor recíproco $(1 / A)$ del número de presencias, sustituidas por el valor individual (110) de los subcuadrantes en los que está presente. El valor $n_{\mathrm{c}}$ corresponde al número de especies presentes en los subcuadrantes en los que estuvo presente la especie $i$. También el índice $I_{c}$ se utilizó como parámetro para cuantificar el grado de rareza o endemismo (mayor o menor restricción espacial) relativo a los muestreos en Cuadrante Tolimán. A medida que sea mayor el índice de área promedio, más grande es el tamaño del área de distribución geográfica (restricción espacial baja), mientras que los valores menores corresponden a una distribución geográfica pequeña (restricción espacial alta).

\section{Complementariedad}

Este concepto se fundamenta en que el contingente regional de especies se encuentra repartido entre diversos puntos o áreas menores que albergan su propia diversidad local, de manera que algunas de ellas sólo se encuentran en ciertas áreas y están ausentes en otras. Así, las áreas menores que presentan especies diferentes son complementarias entre sí, dado que su contribución a la riqueza total es irremplazable. Con este enfoque se aplicó el principio de complementariedad (Vane-Wright et al. 1991; Pressey et al., 1993) a la matriz binaria, simultáneamente con un proceso iterativo de análisis de complementariedad (Margules et al., 1988). Este procedimiento consta de dos etapas básicas: en la primera, se selecciona el subcuadrante con la mayor riqueza de especies y éste constituye el primer sitio o área prioritaria. En la segunda, el total de especies ya seleccionadas se eliminan de la matriz y de este inventario remanente (complemento) se elige el subcuadrante con la mayor riqueza y se integra en segundo lugar en la jerarquía de áreas prioritarias. El procedimiento iterativo continúa de la misma forma para determinar la prioridades sucesivas en la jerarquía (tercera, cuarta y así sucesivamente) y finaliza hasta que todas las especies quedan integradas en la lista de sitios prioritarios para la conservación. Para este propósito se utilizaron dos criterios de selección: (a) la riqueza de especies y (b) el grado de restricción espacial o endemismo.

Recambio de especies (diversidad beta)

Como medida de la diversidad beta entre los subcuadrantes se utilizó el índice de complementariedad (Colwell y Coddington, 1994), que determina el grado de 
heterogeneidad (disimilitud) en la composición de especies entre sitios mediante la siguiente fórmula:

$$
C=\left[\left(S_{j}+S_{k}\right)-2 V_{j k} /\left(S_{j}+S_{k}\right)-V_{j k}\right]^{*} 100
$$

donde $\mathbf{S}_{\mathrm{j}}$ y $\mathbf{S}_{\mathrm{k}}$ son los números de especies en los sitios $\mathbf{j}$ y $\mathbf{k}$, respectivamente, y $\mathbf{V}_{\mathrm{jk}}$ es el número de especies compartidas. Los valores de este índice varían entre $0 \%$ cuando ambos sitios son idénticos en la composición de especies y $100 \%$ cuando son listados absolutamente distintos. Se obtuvo una medida de beta promedio $(\beta m)$ por subcuadrante utilizando las estimaciones parciales de heterogeneidad entre los transectos que integraron cada uno de ellos. Los valores obtenidos de diversidad beta se agruparon en tres categorías porcentuales: (a) 0-33, (b) 34-66 y (c) 67-100. Se considera que porcentajes $>66 \%$ representan un recambio de especies alto (Sánchez y López, 1988).

Clasificación numérica de las muestras florísticas (subcuadrantes)

Los registros florísticos se analizaron mediante el algoritmo de análisis de correspondencia de especies indicadoras de dos vías (Twinspan) y se identificaron los grupos generados, así como la similitud/disimilitud existente entre ellos. El análisis se realizó con el programa PC-Ord Versión 4.2 (McCune y Mefford, 1999).

\section{RESULTADOS}

Listado florístico

Se recolectaron 700 ejemplares botánicos que corresponden a una lista florística de 219 especies y 11 subespecies, pertenecientes a 155 géneros y 63 familias de plantas vasculares. Las familias mejor representadas fueron Cactaceae $(23.8 \%)$, Fabaceae (11.6\%), Asteraceae (11.2\%), Agavaceae (4.3\%), Lamiaceae (4.3\%) y Fagaceae $(3.9 \%)$. Los arbustos $(33.7 \%)$ fueron la forma de vida predominante, seguida de las hierbas (27.7\%) y los árboles (15.6\%), mientras que entre las cactáceas las formas de vida más frecuentes fueron la globosa (47.2\%) y la arbustiva (14.5\%), (ver Apéndice).

Del total de especies registradas, 24 están incluidas en alguna categoría de la NOM-059 (Anónimo, 2010), siete se consideran amenazadas, cinco en peligro de extinción y 12 sujetas a protección especial; 17 de ellas son cactáceas y siete corresponden a otras familias botánicas. Un total de 23 especies son endémicas de 
la Zona Árida Queretano Hidalguense, otras seis están restringidas al Semidesierto Queretano y 22 son propias del Desierto Chihuahuense. Adicionalmente, 14 representan endemismos regionales que abarcan tanto la zona árida como la templada de los estados de Querétaro, Guanajuato y San Luis Potosí. Por su parte, 78 especies están registradas en las colecciones de plantas vivas del Jardín Botánico Regional de Cadereyta "Ing. Manuel González de Cosío", son una representación de la flora regional y sus ejemplares proceden de la misma zona en la que se efectuó el estudio.

Índice de área promedio

Cerca de $30 \%$ de las especies presentaron una distribución geográfica muy pequeña, lo que indica una alta restricción espacial. Esta fracción confinada en áreas reducidas, es también el mayor componente de rareza de todo el contingente de plantas hasta ahora registradas en el Cuadrante Tolimán. La porción restante de especies exhibió valores superiores al promedio del índice de área (Fig. 2), lo cual denota una distribución geográfica más amplia y, en consecuencia, una restricción espacial baja. El patrón general del grado de rareza (restricción espacial) está significativamente correlacionado $(r=0.43, P<0.03)$ con el aumento en la riqueza de especies.

Por otra parte, hay una vinculación positiva y significativa entre la riqueza cactológica y la diversidad general del Cuadrante Tolimán $(r=0.48, P<0.01)$. Como

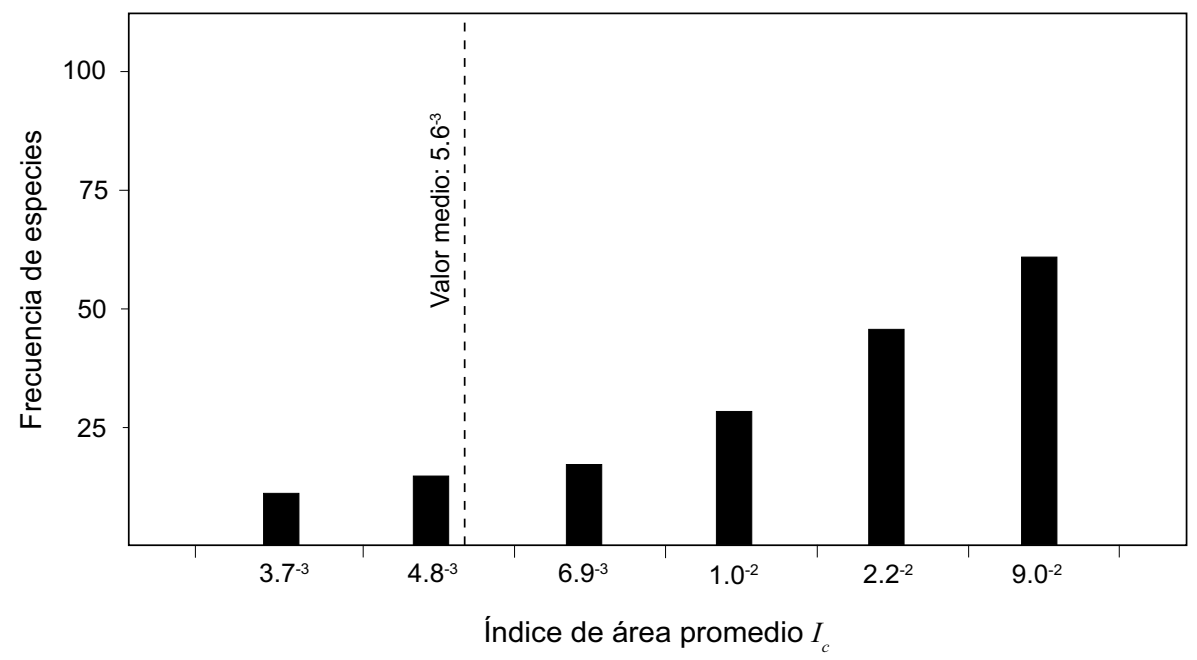

Fig. 2. Índice de área promedio de las especies registradas en el Cuadrante Tolimán. La línea vertical punteada señala la media de $I_{c}$ 
consecuencia de esta asociación, existe también una fuerte correlación positiva en la restricción espacial (rareza) de estos conjuntos florísticos $(r=0.95, P<0.001)$.

Complementariedad y diversidad beta

Los subcuadrantes de mayor importancia para la conservación con base en la riqueza de especies y el grado de endemismo observados se muestran en el Cuadro 1 y la Fig. 3. Este análisis indica que con $1000 \mathrm{~km}^{2}$ (nueve subcuadrantes) se protegería el conjunto de prioridades que definen los principales centros de riqueza y endemismo del Cuadrante Tolimán: zona de Mesa de León, Cadereyta al este (subcuadrante 10; Fig. 1) y una amplia franja-corredor que incluye desde la zona de El Chilar, Tolimán al oeste y la sierra de El Doctor (Cadereyta-San Joaquín), en la Cuenca del río Estórax, en el norte de la región (subcuadrantes 11, 19, 20, 22-25; Fig. 1); junto con un subcuadrante relativamente aislado (el Núm. 1). La diversidad beta (Fig. 3) en los subcuadrantes resultó con heterogeneidad media (34-66\%) y alta (67-100\%). En esta categoría se ubican los sitios prioritarios para la conservación de acuerdo con la complementariedad.

Clasificación de subcuadrantes y relaciones ecológicas entre unidades espaciales

El análisis de la comunidad mediante Twinspan reconoció ocho grupos florísticos (Fig. 4). En este esquema se observa que una primera separación de grupos ubica en el extremo derecho del dendrograma a los subcuadrantes 15, 19, 20 y 25 , en

Cuadro 1. Subcuadrantes prioritarios para la conservación en el Semidesierto Queretano, de acuerdo con la complementariedad de riqueza de especies y endemismo.

\begin{tabular}{clc}
\hline Prioridad & \multicolumn{1}{c}{ Subcuadrante } & $\begin{array}{c}\text { Complementariedad de } \\
\text { riqueza y endemismo (\%) }\end{array}$ \\
\hline 1 & 10 (Mesa de León) & 27.2 \\
2 & 25 (San Joaquín) & 16.5 \\
3 & 22 (El Chilar) & 12.6 \\
4 & 11 (Bernal) & 10.9 \\
5 & 23 (Higuerillas) & 9.4 \\
6 & 24 (La Tinaja) & 8.3 \\
7 & 19 (Altamira) & 5.7 \\
8 & 20 (Maconí) & 5.3 \\
9 & 1 (La Tortuga) & 4.1 \\
\hline
\end{tabular}


la región templada subhúmeda de la zona, mientras que el resto se ubica a la mitad y a la izquierda del mismo y corresponden a las comunidades de afinidad xerófila.

Los subcuadrantes 15, 19, 20 y 25 (bloques VI, VII y VIII; Fig. 4) se caracterizan por las comunidades de Juniperus, Quercus, Pinus y Abies distribuidas en la sierra de El Doctor, que es el parteaguas entre las cuencas de los ríos Estórax y Moctezuma y donde se ubica la segunda mayor elevación del estado de Querétaro; corresponden geográficamente a las localidades de Altamira, Los Juárez y Maconí, en el municipio de Cadereyta, y a la cabecera municipal de San Joaquín, respectivamente. En esta zona la altitud va de 2500 a $3050 \mathrm{~m}$, la temperatura media anual es de aproximadamente $12{ }^{\circ} \mathrm{C}$ y la precipitación total anual oscila entre 600 y $1000 \mathrm{~mm}$.

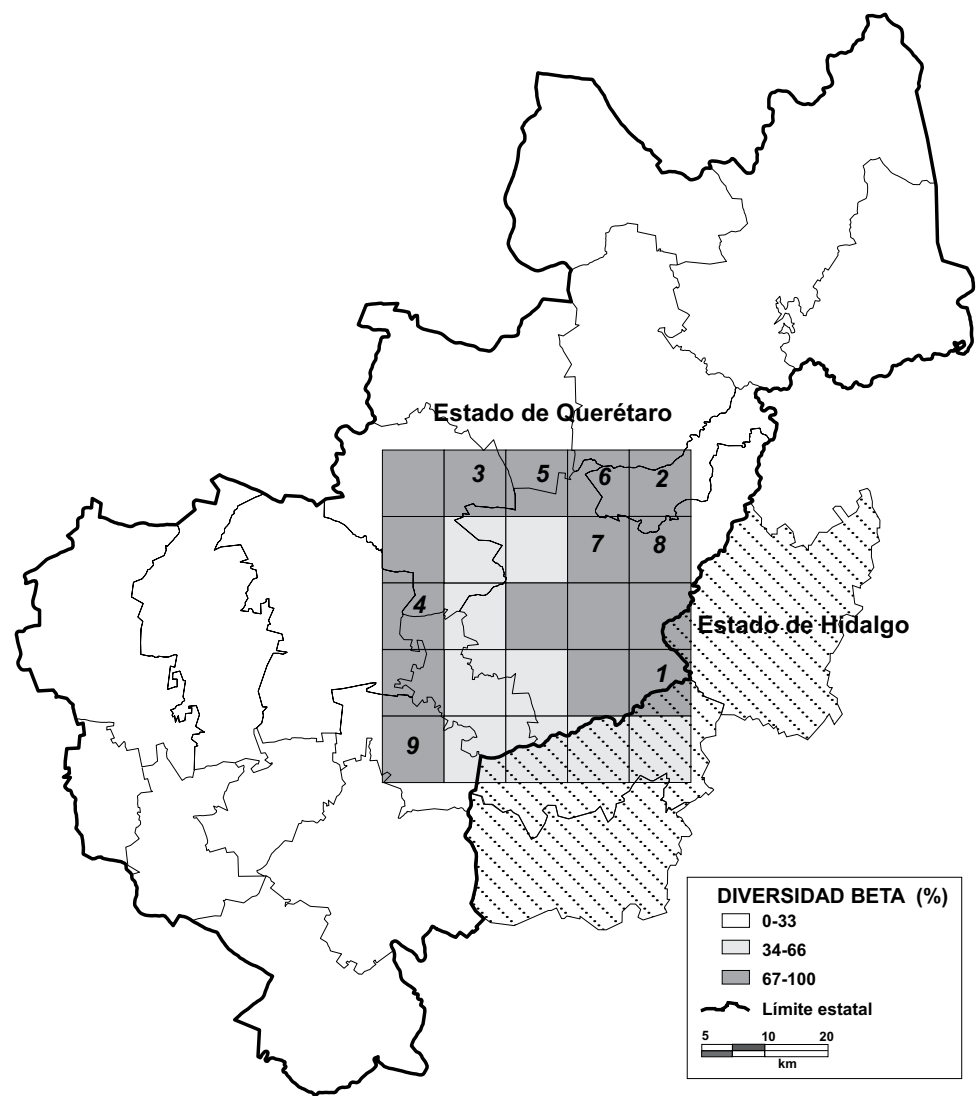

Fig. 3. Sitios prioritarios (1 a 9) para la conservación con base en la complementariedad y el patrón de recambio de especies (diversidad beta) en el Cuadrante Tolimán. 
Los restantes subcuadrantes se acomodan a lo largo de un gradiente de comunidades vegetales xerófilas, en cuyo extremo de mayor humedad se encuentran el encinar arbustivo (matorral esclerófilo) y el matorral submontano. Esta fracción del gradiente de aridez la constituye el subcuadrante 1 (bloque I) que corresponde a las localidades de Santillán, San José de la Laja, La Trinidad y Fuentezuelas, en la porción montañosa del municipio de Tequisquiapan. En esta zona la altitud va de 1900 a $2250 \mathrm{~m}$, la temperatura media anual es de cerca de $18^{\circ} \mathrm{C}$ y la precipitación anual es de alrededor de $500 \mathrm{~mm}$.

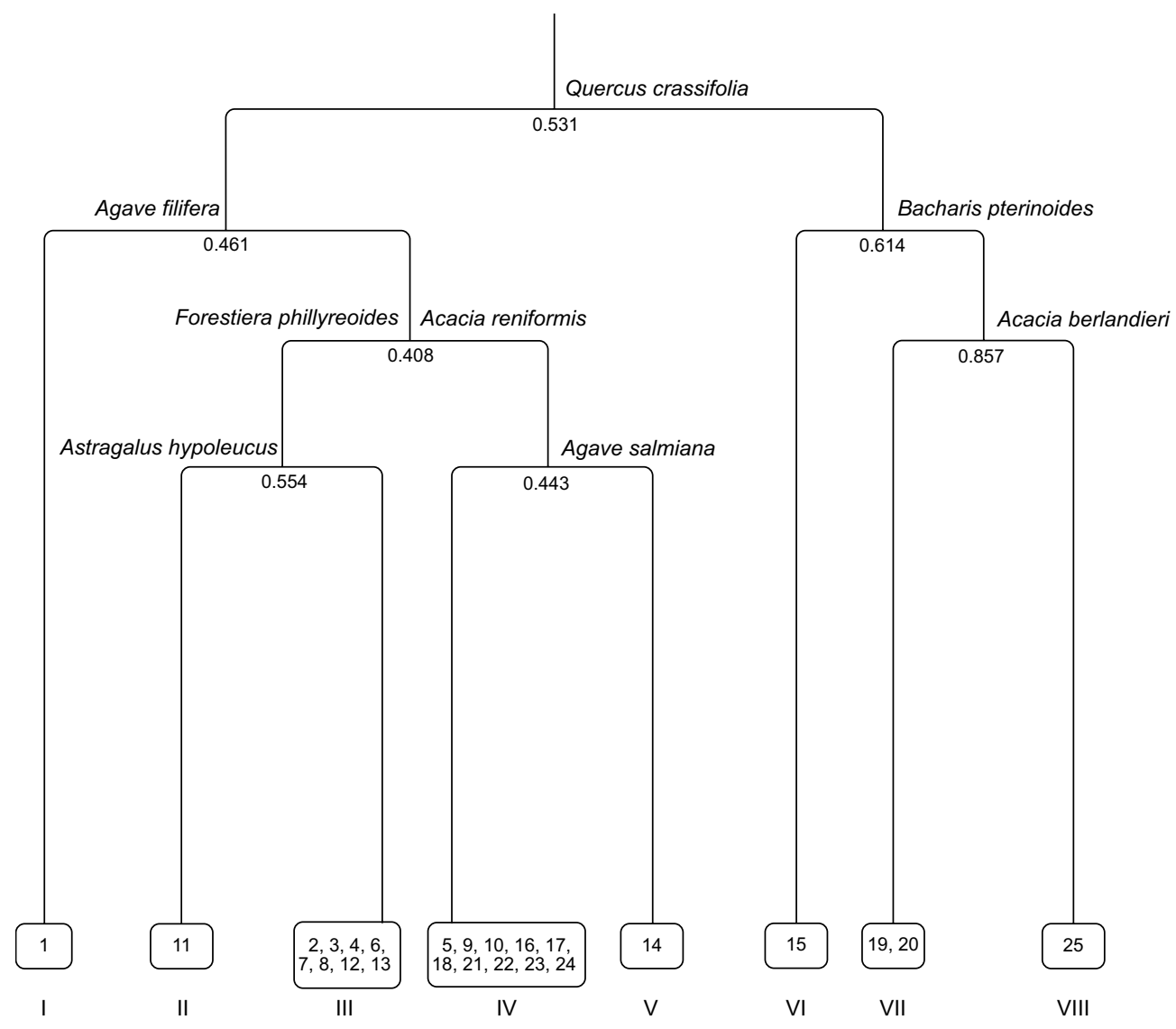

Fig. 4. Clasificación por el método Twinspan de los subcuadrantes que albergan una muestra de la flora del Cuadrante Tolimán. En cada división se muestran las especies indicadoras de uno o ambos lados. Las cifras debajo de cada división son los valores propios (eigenvalues). 
La fracción más seca del área de estudio la conforman todos los demás subcuadrantes, excepto el número 11 (bloque II) que representa una condición ligeramente distinta, pues se trata de una zona en la que coexisten tanto el matorral submontano como el matorral xerófilo micrófilo en un espacio geográfico relativamente reducido determinado por la elevación del cerro San Martín. En esta zona la altitud conforma un gradiente altitudinal de entre 1850 y $2500 \mathrm{~m}$, en el que la temperatura media anual en su parte más baja es de $18{ }^{\circ} \mathrm{C}$ y de $16^{\circ} \mathrm{C}$ en la más alta, mientras que la precipitación anual es de alrededor de $500 \mathrm{~mm}$ en toda la zona.

En cuanto a los tres bloques restantes, el subcuadrante 14 (bloque V), corresponde con la distribución del matorral rosetófilo (Sombrerete, Cadereyta y áreas adyacentes), el cual tiene una composición florística particular y una distribución asociada a sitios montañosos de entre 2000 y $2500 \mathrm{~m}$ de altitud, con una temperatura media anual de $14{ }^{\circ} \mathrm{C}$ y una precipitación anual de alrededor de 650 $\mathrm{mm}$, lo que representa una aridez relativamente menor a la del resto de los sitios xerófilos.

Por otra parte, el bloque III corresponde al matorral crasicaule que se distribuye en el área volcánica de la zona de estudio (subcuadrantes 2, 3, 4, 6, 7, 8, 12, 13), en los alrededores de las cabeceras municipales de Tequisquiapan y Cadereyta. En esta zona la altitud fluctúa entre 1950 y $2400 \mathrm{~m}$, con una temperatura media anual de 15 a $18{ }^{\circ} \mathrm{C}$ y la precipitación anual cercana a los $500 \mathrm{~mm}$.

Finalmente el bloque IV representa a los matorrales crasicaule y micrófilo (subcuadrantes 5, 9, 10, 16, 17, 18, 21, 22, 23, 24), sobre terrenos de roca sedimentaria con altitudes de entre 1450 y $1900 \mathrm{~m}$ sobre el nivel del mar, en donde la temperatura media anual oscila entre $\operatorname{los} 16^{\circ} \mathrm{C}$ y $\operatorname{los} 18^{\circ} \mathrm{C}$, y la precipitación media anual es de menos de $500 \mathrm{~mm}$. Estos matorrales concentran la diversidad más alta y la mayoría de los endemismos de cactáceas del Cuadrante Tolimán, las cuales se distribuyen principalmente en las regiones de Mesa de León y El Chilar, en los municipios de Cadereyta y Tolimán, respectivamente. La primera de ellas en la cuenca del Río Moctezuma, y la segunda en la del Río Estórax. En el Cuadro 2 se presenta un resumen de las condiciones ambientales predominantes en las áreas correspondientes a los bloques de subcuadrantes.

El análisis canónico de correspondencias (ACC) mostró que del total de variables ambientales incluidas en el modelo, cuatro son las relevantes $(P=0.01$, de acuerdo con la prueba de permutación de Monte Carlo) que explican 45.7\% (en los primeros dos ejes de ACC) de la variación total de los datos de las especies (comunidad) en los subcuadrantes (Fig. 5). Se observa un gradiente de aridez, ubicando en el lado izquierdo del gráfico los subcuadrantes más secos y cálidos, y en el derecho 
Cuadro 2. Características ambientales del Cuadrante Tolimán de acuerdo con la clasificación Twinspan.

\begin{tabular}{|c|c|c|}
\hline Bloque & Sub-cuadrantes & Condiciones ambientales \\
\hline $\mathrm{I}$ & $\begin{array}{l}1 \\
\text { Santillán, San José de } \\
\text { la Laja, La Trinidad y } \\
\text { Fuentezuelas, Mpio. } \\
\text { Tequisquiapan. }\end{array}$ & $\begin{array}{l}\text { Sierra de Tequisquiapan. Basamento geológico } \\
\text { de riolita perteneciente a la provincia fisiográfica } \\
\text { Mesa del Centro. Altitud entre } 1900 \text { y } 2250 \text {; } \\
\text { temperatura media anual de aproximadamente } 18 \\
{ }^{\circ} \mathrm{C} \text { y precipitación anual de ca. } 500 \mathrm{~mm} \text {. }\end{array}$ \\
\hline II & $\begin{array}{l}11 \\
\text { Sub-cuadrante Bernal, } \\
\text { Mpios. Ezequiel } \\
\text { Montes y Tolimán. }\end{array}$ & $\begin{array}{l}\text { Cerro San Martín. Zona de confluencia entre las } \\
\text { provincias fisiográficas Mesa del Centro (riolitas) } \\
\text { y Sierra Madre Oriental (Caliza). Altitud de } 1850 \text { a } \\
2500 \mathrm{~m} \text {. Temperatura media anual de entre } 16 \text { y } 18 \\
{ }^{\circ} \mathrm{C} \text { dependiendo de la altitud, y precipitación anual } \\
\text { de ca. } 500 \mathrm{~mm} \text {. }\end{array}$ \\
\hline
\end{tabular}

III

2, 3, 4, 6, 7, 8, 12, 13 Basamento de rocas volcánicas en la confluencia Cabeceras municipales de las provincias fisiográficas Mesa del Centro y de Tequisquiapan, Eje Neovolcánico. Altitud entre 1950 y 2400 m. Cadereyta, Qro. y Temperatura media anual de entre 15 y $18^{\circ} \mathrm{C}$ y parte de Tecozautla, precipitación anual de ca. $500 \mathrm{~mm}$. Hidalgo.

\begin{tabular}{|c|c|c|}
\hline IV & $\begin{array}{l}\text { 5, 9, 10, 16, 17, } 18, \\
\text { 21, 22, 23, } 24 \\
\text { Mesa de León, } \\
\text { Cadereyta y El Chilar, } \\
\text { Tolimán. }\end{array}$ & $\begin{array}{l}\text { Basamento de roca sedimentaria (caliza del } \\
\text { Cretácico Superior). Altitud de entre } 1450 \text { y } \\
1900 \mathrm{~m} \text {, temperatura media anual de } 16 \text { a } 18^{\circ} \mathrm{C} \mathrm{y} \\
\text { precipitación anual de menos de } 500 \mathrm{~mm} .\end{array}$ \\
\hline V & $\begin{array}{c}14 \\
\text { Sombrerete, } \\
\text { Cadereyta. }\end{array}$ & $\begin{array}{l}\text { Laderas y pie de monte de la sierra El Doctor. } \\
\text { Basamento de roca caliza del Cretácico Superior, } \\
\text { perteneciente a la provincia fisiográfica de la Sierra } \\
\text { Madre Oriental; altitud entre } 2000 \text { y } 2500 \mathrm{~m} \text {. } \\
\text { Temperatura media anual de alrededor de } 14{ }^{\circ} \mathrm{C} \text { y } \\
\text { precipitación anual de ca. } 650 \mathrm{~mm} \text {. }\end{array}$ \\
\hline VI, VII, VIII & $\begin{array}{l}15,19,20 \text { y } 25 \\
\text { Altamira, Los Juárez } \\
\text { y Maconí, Cadereyta, } \\
\text { y San Joaquín, Mpio. } \\
\text { San Joaquín. }\end{array}$ & $\begin{array}{l}\text { Sierra El Doctor. Basamento de roca caliza del } \\
\text { Cretácico Superior, perteneciente a la provincia } \\
\text { fisiográfica de la Sierra Madre Oriental; altitudes } \\
\text { entre } 2500 \text { y } 3050 \mathrm{~m} \text { s.n.m., Temperatura media } \\
\text { anual de aproximadamente } 12^{\circ} \mathrm{C} \text { y precipitación } \\
\text { total anual entre } 600 \text { y } 1000 \mathrm{~mm} \text {. }\end{array}$ \\
\hline
\end{tabular}


los más húmedos y templados, así como los subcuadrantes asociados a una mayor altitud y un relieve más accidentado.

\section{DISCUSIÓN}

Desde una perspectiva fitogeográfica el Semidesierto Queretano se ha reconocido como una región con abundancia de especies endémicas locales, principalmente de afinidad xerófila; por otra parte, se considera que a nivel florístico el Semidesierto

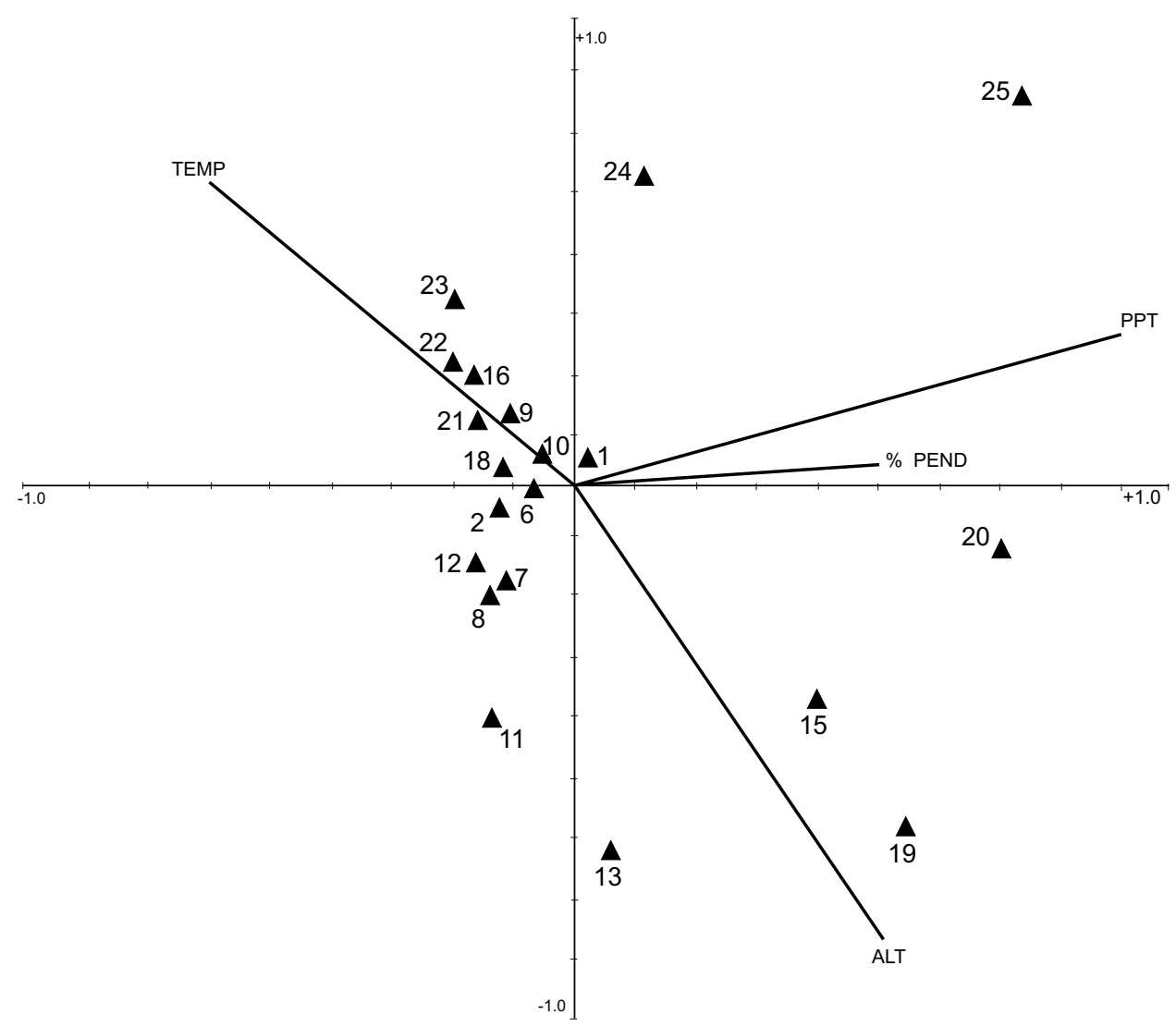

Fig. 5. Análisis canónico de correspondencia (ACC) de las variables ambientales relevantes (vectores: TEMP $=$ temperatura media anual, $\mathrm{PPT}=$ precipitación media anual, $\% \mathrm{PEND}=\%$ de pendiente y ALT $=$ altitud) y los subcuadrantes $(\mathbf{\Lambda})$ en el Cuadrante Tolimán. 
Queretano y las zonas xerófilas del estado de Hidalgo están estrechamente relacionadas y que en conjunto conforman la Zona Árida Queretano Hidalguense, la cual se ubica en la porción sur extrema del Desierto Chihuahuense (Zamudio, 1984). Sin embargo, se piensa que debido a la disyunción de esta zona con respecto al cuerpo principal de este último (Medellín, 1982), tal aislamiento le confiere un papel de refugio o el carácter de "relictual" para varias especies xerófilas de esta vasta región, y se especula que tales albergues posiblemente se generaron en eventos glaciales del Pleistoceno (Zamudio, 1984), aunque las evidencias fósiles sugieren que probablemente esta discontinuidad espacial se produjo desde el Holoceno (Rzedowski y Calderón de Rzedowski, 1988). También se ha postulado la existencia de refugios de plantas y/o paleoendemismos (Rzedowski, 1966; Medellín, 1982; Zamudio, 1984) en otras regiones del Desierto Chihuahuense. Particularmente se señala el hecho de que esta eco-región posee elementos florísticos que lo hacen sustancialmente diferente de otras grandes regiones secas del territorio mexicano, como el Desierto de Sonora y el Valle de Tehuacán-Cuicatlán (Rzedowski, 1973). En este contexto, los estudios previos en la comarca (Zamudio, 1984; Fernández y Colmenero, 1997) señalan la existencia de complejos gradientes ambientales que sustentan mosaicos variados de vegetación en espacios relativamente pequeños, los cuales expresan una alta diversidad de especies, y que en la zona se traduce en una elevada diversidad beta.

Aunque el Cuadrante Tolimán es una delimitación espacial arbitraria y por lo tanto no es una región con fronteras naturales propiamente dichas, sí expresa los principales rasgos florísticos y ambientales del Semidesierto Queretano, de reconocida particularidad biogeográfica (Zamudio, 1984; Zamudio et al., 1992; Scheinvar, 2005). El mayor recambio de especies tiene lugar en las zonas de transición que forman los profundos cañones que integran la cuenca de los ríos Estórax y Moctezuma y las áreas templadas montañosas del macizo El Doctor, fenómeno que se podría atribuir a la afinidad de muchas plantas por áreas cálido-secas por un lado, y por áreas templadosecas y templado-subhúmedas por el otro, así como a los ecotonos resultantes entre ambas condiciones. Por ejemplo, la presencia de los cinturones de niebla que circundan la sierra del Doctor en franjas altitudinales de entre 1000 y 2200 m (Martorell y Ezcurra, 2002) están asociados al matorral submontano, comunidad vegetal ecotonal que se establece entre los matorrales xerófilos y los bosques templados. El matorral submontano es el hábitat de especies amenazadas como Dasylirion acrotrichum, D. longissimum y de otras de distribución muy restringida y en riesgo de extinción como Mammillaria herrerae y Thelocactus hastifer. En general, las partes con la mayor diversidad beta en el Cuadrante Tolimán son la que integran un corredor casi perimetral que coincide con la delimitación natural del Semidesierto Queretano en sus 
bordes oriente y poniente, pero no en sus límites norte y sur, donde es más extenso. Estas áreas, al igual que las de mayor complementariedad son, en términos de riqueza, las prioritarias para la conservación.

Con el enfoque de complementariedad utilizado se optimizó la selección de sectores para la conservación, dado que a) se identificaron los sitios complementarios que integraron la mayor proporción de especies y b) se redujo el número de subcuadrantes para conservar. Es decir, se conjuntaron simultáneamente las áreas de alta complementariedad y de alta riqueza y endemismo, lo cual determinó los sitios prioritarios para la conservación. Con criterios similares se determinó jerárquicamente el orden de prioridad de los sitios seleccionados para la conservación de las áreas (subcuadrantes) que conforman la región.

Con este procedimiento la selección de áreas para la conservación puede tener mayores posibilidades de ser efectiva, ya que (a) existe una idea clara de hacia dónde dirigir y concentrar los esfuerzos de conservación, y (b) permite identificar a las plantas que marcan la diferencia en los valores de complementariedad, las cuales también pueden considerarse como prioritarias; comúnmente tales especies son las de distribución restringida. Así por ejemplo, en términos espaciales la aplicación de este método en el Cuadrante Tolimán sugiere que con la protección de $990 \mathrm{~km}^{2}$ (alrededor de 40\% del área total) se tendría representado el acervo biológico del Semidesierto Queretano, ya que esta superficie integra las zonas que contribuyen sustancialmente a la diversidad y el endemismo de la región. El método destaca particularmente dos áreas o núcleos de riqueza y endemismo bien definidas: hacia el noroeste (cuenca del Estórax) y el sureste (cuenca del río Moctezuma) del Cuadrante Tolimán.

Dado el esquema observado del índice de área geográfica promedio, el patrón de distribución de la riqueza y el endemismo sugiere que: (a) los sitios con la mayor riqueza florística albergan significativamente más especies endémicas o con áreas de distribución reducida, por lo tanto, la rareza se encuentra anidada en los lugares de mayor diversidad, y (b) el componente endémico a nivel local, más que el regional, es el que genera dicho patrón. Entre los endemismos locales, la contribución más importante está dada por las cactáceas y ocurre un patrón semejante: los subcuadrantes con la mayor diversidad cactológica contienen significativamente más especies raras. Esto implica que la conservación de áreas con elevada rareza de especies o un endemismo alto tendría un mayor impacto en términos de protección regional de la riqueza de especies.

Tanto la complementariedad (diversidad beta) como el índice de área promedio son métodos convergentes que facilitan la selección de zonas prioritarias para 
la conservación cuando se busca destacar el endemismo local y regional, pero sin ignorar a los elementos florísticos comunes.

El arreglo espacial de la clasificación de subcuadrantes denota las grandes asociaciones vegetales de la región, las cuales mostraron una gran heterogeneidad, lo que sugiere una variación amplia en la composición de especies, aun en el último proceso divisivo. Este rasgo podría ser indicativo de que a pesar de ser una región predominantemente seca, el gradiente altitudinal recorrido (de 1400 a 3000 m) implica cambios de temperatura y precipitación significativos que se reflejan en la composición específica.

Los subcuadrantes incluidos en un grupo (área) no aparecen muy dispersos o inconexos, de modo que representan áreas más o menos gruesas de vegetación definida que se apegan a lo observado. Por ejemplo, la denominación "matorral xerófilo" incluye al menos cinco variantes de este tipo de vegetación (sensu Zamudio et al., 1992), lo cual encierra una compleja combinación de comunidades y asociaciones, formas de vida y ecotonos relativamente amplios. Una situación semejante de variación se presenta en las comunidades afines a los bosques templados. Finalmente, esta condición se expresa en el inventario registrado, el cual resultó relativamente diferente de otros listados florísticos realizados (Zamudio, 1984; Fernández y Colmenero, 1997; Zamudio et al., 1992).

Para algunas zonas del sur del Desierto Chihuahuense que sustentan una alta riqueza florística (Huerta-Martínez y García-Moya, 2004) y cactológica (Hernández et al., 2001) se ha sugerido que hay una estrecha relación de estos contingentes florísticos con gradientes ambientales, tanto a la escala del paisaje como a la de nivel local (Huerta-Martínez et al., 2004). Sobre el particular, los gradientes detectados con relación a la riqueza florística sugieren que (a) la variación de las comunidades vegetales está dada parcialmente por la heterogeneidad ambiental de la región, (b) los gradientes de precipitación, temperatura y altitud favorecen una gran variedad de condiciones ambientales; por ejemplo, la amplitud del gradiente de aridez se refleja en la presencia de comunidades vegetales que van desde las condiciones xérico-secas (termófilas) hasta las xérico-templadas (mesófilas), y (c) a estas variadas combinaciones de xerófitas se añade el gradiente altitudinal, el cual contribuye notoriamente a la heterogeneidad espacial de la región (por ejemplo, la presencia de profundos cañones en el río Estórax desde 600 m s.n.m. hasta elevaciones mayores de $3000 \mathrm{~m}$ en la sierra de El Doctor).

La influencia de la historia natural reciente es un elemento importante a considerar en la florística del Semidesierto Queretano. Por ejemplo, con las Cactaceae se ha argumentado sobre la convergencia de procesos histórico-biogeográficos y 
ecológicos para explicar sus patrones de riqueza y endemismo (Hernández y Bárcenas, 1995, Riddle et al., 2000; Hernández et al., 2001). La hipótesis basada en la influencia de los cambios climáticos del Pleistoceno en la conformación actual de la vegetación del Desierto Chihuahuense, incluidas las Cactaceae, es la que mayor atención ha recibido (Hillesheim et al., 2005). Hay evidencias de que la dinámica climática pleistocénica produjo condiciones diversas en los últimos 11,000 años, alternando ciclos secos-cálidos, secos fríos y cálido-húmedos (Ortega-Ramírez et al., 1998; Waters y Vance, 2001; Musgrove et al., 2001), presumiblemente ejerciendo efectos de contracción, extensión y aislamiento geográfico en varios taxa (Hernández y Bárcenas, 1996). Así, con la aplicación del índice de área promedio se reconoció que los endemismos del Cuadrante Tolimán se restringen a áreas muy limitadas, fenómeno que no es raro en el Desierto Chihuahuense (Hernández et al., 2004), pero se desconocen aspectos paleoambientales con alguna implicación en el componente endémico de la región.

En virtud del conocimiento florístico previo acerca del Semidesierto Queretano, cabría esperar que hubiera una correspondencia lógica entre la riqueza de cactáceas y la riqueza de la flora que coexiste con este grupo; sin embargo, este patrón no debe considerarse causal, dado que relaciones semejantes se han observado en otras regiones dentro del Desierto Chihuahuense (Huerta-Martínez y García-Moya, 2004; Martínez-Ávalos y Jurado, 2005; González et al., 2007) y fuera de esta eco-región (Dávila et al., 2002), lo cual sugiere su importancia como elementos para la conservación biológica de las zonas áridas y semiáridas. No obstante esta situación, actualmente el Semidesierto Queretano carece de protección formal y legal. En contraparte, se conoce un número elevado y creciente de factores de perturbación antrópica (minería, obras hidráulicas, disturbio crónico de las comunidades) que podrían tener fuertes impactos negativos sobre la flora que alberga esta región.

\section{AGRADECIMIENTOS}

Una parte de este trabajo fue auspiciado a través del proyecto QRO-2003-CO110152 -CONACYT-Fondos Mixtos-Gobierno del estado de Querétaro. Las colectas de material vegetal fueron hechas con la autorización de SEMARNAT mediante los permisos FLOR-0100, FLOR 0166, SGPA/DGVS/03337 y SGPA/DGVS/05915. Los árbitros anónimos hicieron importantes sugerencias para la versión final del manuscrito. 


\section{LITERATURA CITADA}

Anderson, S. 1994. Area and endemism. Quart. Rev. Biol. 69: 451-471.

Anónimo. 1986. Síntesis geográfica, noménclator y anexo cartográfico del Estado de Querétaro. Secretaría de Programación y Presupuesto. México, D.F., México. 141 pp.

Anónimo. 2000. Carta de uso de Suelo, escala 1: 250,000 (F1410). Instituto Nacional de Estadística Geografía e Informática. Aguascalientes, Aguascalientes, México.

Anónimo. 2010. Norma Oficial Mexicana NOM-059-SEMARNAT-2010. Protección ambiental-especies nativas de México de flora y fauna silvestres-categorías de riesgo y especificaciones para su inclusión, exclusión o cambio-lista de especies en riesgo. Secretaría del medio ambiente y recursos naturales, Diario Oficial de la Federación. 30 de diciembre de 2010. http://dof.gob.mx/nota_detalle.php?codigo=5173091\& fecha $=30 / 12 / 2010$

Argüelles, E., R. Fernández y S. Zamudio. 1991. Listado florístico preliminar del estado de Querétaro. Flora del Bajío y de Regiones Adyacentes II: 155 pp.

Bravo-Hollis, H. 1978. Las cactáceas de México. Tomo I. Universidad Nacional Autónoma de México. México, D.F., México. 743 pp.

Brown, J. 1995. Macroecology. The University of Chicago Press. Chicago, USA. 284 pp.

Chávez, R., E. Sánchez, M. Hernández, J. G. Hernández-Oria y R. Hernández. 2006. Propagación de especies amenazadas de la familia Cactaceae del Semidesierto Queretano. Bol. Soc. Lat. Car. Cact. Suc. 3(2): 9-13.

Colwell, R. K. y J. A. Coddington. 1994. Estimating terrestrial biodiversity through extrapolation. Phil Trans. R. Soc. Lond. B. Biol. Sci. 345: 101-118.

Contreras-Medina, R. 2006. Los métodos de análisis biogeográfico y su aplicación a la distribución de las gimnospermas en México. Interciencia 31: 176-182.

Crovello, Th. J. 1981. Quantitative biogeography: an overview. Taxon 30: 563-575.

Dávila, P., M. Arizmendi, A. Valiente-Banuet, J. L. Villaseñor, A. Casas y R. Lira. 2002. Biological diversity in the Tehuacán-Cuicatlán Valley, México. Biodiv. Cons. 11: 421-442.

Dinerstein, E., D. Olson, J. Atchley, C. Loucks, S. Contreras-Balderas, R. Abell, E. Iñigo, E. Enkerlin, C. E. Williams y G. Castilleja (eds.). 1999. Ecoregion-based conservation in the Chihuahuan Desert: a biological assessment and biodiversity vision. World Wild Fund, Comisión Nacional para el Conocimiento y uso de la Biodiversidad, Pronatura A.C. e Instituto Tecnológico y de Estudios Superiores de Monterrey. Washington, D.C. USA. 489 pp.

Fernández, R. y J. A. Colmenero. 1997. Notas sobre la vegetación y flora del municipio de San Joaquín, Querétaro, México. Polibotánica 4: 10-36.

Gómez-Hinostrosa, C. y H. M. Hernández. 2000. Diversity, geographical distribution and conservation of Cactaceae in the Mier y Noriega region, Mexico. Biodiv. Cons. 9: 403-418.

González, O., J. Giménez, J. García y R. Aguirre. 2007. Flórula vascular de la sierra de Catorce y territorios adyacentes, San Luis Potosí, México. Acta Bot. Mex. 78: 1-38. 
Guzmán, U., S. Arias y P. Dávila. 2003. Catálogo de cactáceas mexicanas. Universidad Nacional Autónoma de México y Comisión Nacional para el Conocimiento y Uso de la Biodiversidad. México, D.F., México. 315 pp.

Hernández, H. M. y R. T. Bárcenas. 1995. Endangered cacti in the Chihuahuan Desert: I. Distribution patterns. Cons. Biol. 9: 1176-1188.

Hernández, H. M. y R. T. Bárcenas. 1996. Endangered cacti in the Chihuahuan Desert: II. Biogeography and Conservation. Cons. Biol. 10: 1200-1209.

Hernández,H. M., C. Gómez-Hinostrosa y R. T. Bárcenas. 2001. Diversity, spatial arrangement and endemism of Cactaceae in the Huizache area, a hot spot in the Chihuahuan Desert. Biodiv. Cons. 10: 1097-1112.

Hernández, H. M., C. Gómez-Hinostrosa y B. Goettsch. 2004. Checklist of Chihuahuan Desert Cactaceae. Harv. Pap. Bot. 9: 51-68.

Hernández-Oria, J. G., R. Chávez y E. Sánchez. 2007b. Factores de riesgo en las Cactaceae amenazadas de una región semiárida en el sur del Desierto Chihuahuense, México. Interciencia 32: 728-734.

Hillesheim, M., D. Hodell, B. Leyden, M. Brenner, J. Curtis, F. Anselmetti, D. Ariztegui, D. Buck, T. Guilderson, M. Rosenmeier y W. Schnurrenberger. 2005. Climate change in lowland Central America during the late deglacial and early Holocene. J. Quat. Sci. 20: 363-376.

Huerta-Martínez, F. y E. García-Moya. 2004. Diversidad de especies y su relación con el ambiente en un área semiárida del centro de México: implicaciones para la conservación. Interciencia 29: 435-444.

Huerta-Martínez, F., J. A. Vázquez-García, E. García-Moya, L. López-Mata y H. VaqueraHuerta. 2004. Vegetation ordination at the southern Chihuahuan Desert (San Luis Potosí, México). Plant Ecol. 174: 79-87.

Hunt, D. R. 1999. Cites Cactaceae checklist. Royal Botanic Gardens. Kew, UK. 190 pp.

Jongman, R. H. G, C. J. F. Ter Braak y O. F. R. Van Tongeren. 2000. Data analysis in community and landscape ecology. Cambridge University Press. Cambridge, UK. 229 pp.

Kovach, W. L. 1999. MVSP-A multivariate statistical package for Windows ver. 3.1. Kovach Computing Services. Pentraeth, UK. 133 pp.

Margules, C. R., A. O. Nicholls y R. L. Pressey. 1988. Selecting networks of reserves to maximise biological diversity. Biol. Cons. 43: 63-76.

Martínez-Ávalos, J. G. y E. Jurado. 2005. Geographic distribution and conservation of Cactaceae from Tamaulipas, Mexico. Biodiv. Cons. 14: 2483-2506.

Martorell, C. y E. Ezcurra. 2002. Rosette scrub occurrence and fog availability in arid mountains of Mexico. J. Veg. Sci. 13: 651-662.

McCune, B. y M. J. Mefford. 1999. PC-Ord: Multivariate analysis of ecological data. Versión 4.2. MjM Software Design. Gleneden Beach, Oregon, USA.

Medellín, F. 1982. The Chihuahuan Desert. In: Bender, G. L. (ed.). Reference handbook on the deserts of North America. Greewood Press. Westport, Connecticut, USA. pp. 321-381.

Méndez-Larios, I., E. Ortiz y J. L. Villaseñor. 2004. Las Magnoliophyta endémicas de la porción xerofítica de la provincia florística del valle de Tehuacán-Cuicatlán, México. An. Inst. Biol. Univ. Nal. Aut. México, Ser. Bot. 75: 87-104. 
Méndez-Larios, I., J. L. Villaseñor, R. Lira, J. J. Morrone y E. Ortiz. 2005. Toward the identification of a core zone in the Tehuacán-Cuicatlán Biosphere Reserve, Mexico, based on parsimony analysis of endemicity of flowering plant species. Interciencia 30(5): 267-274.

Musgrove, M., J. Banner, L. Mack, D. Combs, E. James, H. Cheng y L. Edwards. 2001. Geochronology of late Pleistocene to Holocene speleothems from Central Texas: Implications for regional Paleoclimate. Geol. Soc. Am. Bull. 113: 1532-1543.

Ortega-Ramírez, J., A. Valiente-Banuet, J. Urrutia-Fucugauchi, C. Mortera-Gutiérrez y G. Alvarado-Valdez. 1998. Paleoclimatic changes during the Late Pleistocene-Holocene in Laguna Babícora, near the Chihuahuan Desert, México. Can. J. Earth Sci. 35: 11681179.

Pressey, R. L., C. J. Humpries, R. Margules, I. Vane-Wright y P. H. Williams. 1993. Beyond opportunism: key principles for systematic reserve selection. Trends Ecol. Evol. 8: 124-128.

Rapoport, E. H. y A. Monjeau. 2001. Areografía. In: Llorente, J. y J. J. Morrone (eds.). Introducción a la biogeografía en Latinoamérica: teorías, conceptos, métodos y aplicaciones. Universidad Nacional Autónoma de México. México, D.F. México. pp. 23-30.

Riddle, B. R., D. J. Hafner y L. F. Alexander. 2000. Phylogeography and systematics of the Peromyscus eremicus species group and the historical biogeography of North American warm regional deserts. Mol. Phyl. Evol. 17: 145-160.

Rzedowski, J. 1966. Vegetación del estado de San Luis Potosí. Acta Cient. Pot. Méx. 5: 1-291.

Rzedowski, J. 1973. Geographical relationship of the flora of Mexican dry regions. In: Graham, A. (ed.). Vegetation and vegetational history of northern Latin America. Elsevier. Amsterdam, Holanda. pp. 61-72.

Rzedowski, J. 1978. Vegetación de México. Ed. Limusa. México, D.F. 432 pp.

Rzedowski, J. 1991. Diversidad y orígenes de la flora fanerogámica de México. Acta Bot. Mex. 14: 3-21.

Rzedowski, J. 2005. México como área de origen y diversificación de linajes vegetales. In: Llorente, J. y J. J. Morrone (eds.). Regionalización biogeográfica en Iberoamérica y tópicos afines. Las Prensas de Ciencias. Universidad Nacional Autónoma de México. México, D.F., México. pp. 375-382.

Rzedowski, J. y G. Calderón de Rzedowski. 1988. Dos nuevas localidades de Larrea tridentata (Zygophyllaceae) en el centro de México y su interés fitogeográfico. Acta Bot. Mex. 1: 7-9.

Sánchez, E., R. Chávez, J. G. Hernández-Oria y M. Hernández. 2006. Especies de Cactaceae prioritarias para la conservación en la zona árida Queretano-Hidalguense. Consejo de Ciencia y Tecnología del estado de Querétaro. Querétaro, Querétaro, México. 97 pp.

Sánchez, O. y G. López. 1988. A theoretical analysis of some indices of similarity as applied to biogeography. Folia Ent. Mex. 75: 119-145.

Santos, M. y H. Arita. 2002. Priority areas for the conservation of New World nectarfeedingbats. In: Fleming, T. y A. Valiente-Banuet (eds.). Columnar cacti and their mutualists. The University of Arizona Press. Tucson, Arizona, USA. pp. 342-363. 
Scheinvar, L. 2005. Flora cactológica del estado de Querétaro. Fondo de Cultura Económica. México, D.F., México. 390 pp.

Vane-Wright, R., C. Humphries y P. H. Williams. 1991. What to protect? Systematics and the agony of choice. Biol. Cons. 55: 235-254.

Villaseñor, J. L. 2003. Diversidad y distribución de las Magnoliophyta de México. Interciencia 28: 160-167.

Villaseñor, J. L. y T. S. Elias. 1995. Análisis de especies endémicas para identificar áreas de protección en Baja California, México. In: Linares, E., P. Dávila, F. Chiang, R. Bye y T. Elias (eds.). Conservación de plantas en peligro de extinción: diferentes enfoques. Instituto de Biología, Universidad Nacional Autónoma de México. México, D.F., México. pp. 43-50.

Villaseñor, J. L., G. Ibarra-Manríquez y D. Ocaña. 1998. Strategies for the conservation of Asteraceae in Mexico. Cons. Biol. 12: 1066-1075.

Waters, M. y C. Vance. 2001. Late Quaternary arroyo formation and climate change in the American Southwest. Geology 29: 399-402.

Williams, P. H., G. Prance, C. J. Humphries y K. S. Edwards. 1996. A comparison of richness hotspots, rarity hotspots and complementarity areas for conserving diversity of British birds. Cons. Biol. 10: 155-174.

Zamudio, S. 1984. La vegetación de la cuenca del río Estórax y sus relaciones fitogeográficas. Tesis de licenciatura. Facultad de Ciencias, Universidad Nacional Autónoma de México. México, D.F., México. 275 pp.

Zamudio, S., J. Rzedowski, E. Carranza y G. Calderón. 1992. La vegetación en el estado de Querétaro. Consejo de Ciencia y Tecnología del estado de Querétaro. Querétaro, Querétaro, México. 92 pp. 


\section{APÉNDICE}

Lista de especies de plantas registradas en el Cuadrante Tolimán. La nomenclatura usada en Cactaceae está de acuerdo con Guzmán et al. (2003), a excepción del género Stenocactus, referido según Hunt (1999). El listado se presenta del siguiente modo: la forma de vida ( $\mathrm{Ar}=$ árbol, ar $=$ arbusto, $\mathrm{h}=$ hierba, ep $=$ epífita, $\mathrm{r}=$ roseta; para las cactáceas se usaron los tipos morfológicos de Bravo-Hollis (1978): $\mathrm{Gt}=$ globosa de tallos hipocotiledonares, $\mathrm{g}=$ globosa, $\mathrm{Cc}=$ cilíndrica cespitosa, $\mathrm{c}=$ cilíndrica, ar $=$ arbustiva, $\mathrm{Ab}=$ arborescente, $\mathrm{Gc}=$ globosa cespitosa), especie, autor, seguido de una abreviatura en paréntesis y en negritas cuando la especie o subespecie está incluida en alguna categoría de la Norma Oficial Mexicana-ECOL059 (Anónimo, 2010), señalados de acuerdo con las siguientes notaciones en negritas: $\mathbf{P r}$ (sujeta a protección especial), A (amenazada), P (en peligro de extinción). Después está anotado el número de colecta y en paréntesis el(los) herbario(s) donde fueron depositados: (1) IEB, (2) QMEX, (3) MEXU, (4) Jardín Botánico Regional de Cadereyta. La abreviatura (JB) denota si la especie está representada en las colecciones del Jardín Botánico Regional de Cadereyta. Finalmente, se indica el nivel de endemismo en los taxa que presentan esta particularidad.

\begin{tabular}{|l|c|c|}
\hline \multicolumn{1}{|c|}{ TAXON } & Núm. de colecta & Amplitud geográfica \\
\hline ar Ephedra compacta Rose & & \\
& $\begin{array}{c}1280,12117 \\
(1,2,3)\end{array}$ & \\
\hline ar Ephedra pedunculata Engelm. & $12226,12150(2)$ & \\
\hline \multicolumn{1}{|c|}{ CUPRESSACEAE } & & \\
\hline Ar Juniperus monosperma var. gracilis Mart. & $12216(2)$ & Sierra Madre Oriental \\
\hline ar Agave americana L. & & \\
\hline ar Agave difformis A. Berger & JB & \\
\hline ar Agave filifera Salm-Dyck & JB & \\
\hline ar Agave gentryi B. Ullrich & JB & \\
\hline ar Agave salmiana Gentry & JB & \\
\hline ar Agave scabra Ortega & 12127 & Zona Árida Queretano \\
\hline ar Agave striata Zucc. subsp. falcata \\
(Engelm.) H. Gentry & JB & \\
\hline ar Agave xylonacantha Salm-Dyck & JB & \\
\hline Ar Yucca filifera Chabaud & JB & \\
\hline Ar Yucca queretaroensis Piña (Pr) & JB & \\
\hline
\end{tabular}


Apéndice. Continuación.

\begin{tabular}{|c|c|c|}
\hline TAXON & Núm. de colecta & Amplitud geográfica \\
\hline \multicolumn{3}{|l|}{ AMARYLLIDACEAE } \\
\hline h Bravoa geminiflora La Llave \& Lex. & $12086(2,4)$ & \\
\hline \multicolumn{3}{|l|}{ ANTHERICACEAE } \\
\hline $\mathrm{r}$ Anthericum nanum Baker & $12037(2,3)$ & \\
\hline r Echeandia flexuosa Greenm. & $12083(2,3)$ & \\
\hline \multicolumn{3}{|l|}{ ARECACEAE } \\
\hline Ar Brahea berlandieri Bartl. (Pr) & $2209(2)$ & \\
\hline \multicolumn{3}{|l|}{ BROMELIACEAE } \\
\hline h Hechtia glomerata Zucc. & JB & \\
\hline \multicolumn{3}{|l|}{ COMMELINACEAE } \\
\hline h Gibasis pulchella (Kunth) Raf. & $12071(1,2,3)$ & \\
\hline \multicolumn{3}{|l|}{ HYACINTHACEAE } \\
\hline r Hemiphylacus latifolius S. Watson & JB & \begin{tabular}{|c|} 
Desierto Chihuahuense, \\
Valle de Tehuacán-Cuicatlán
\end{tabular} \\
\hline \multicolumn{3}{|l|}{ LILIACEAE } \\
\hline $\begin{array}{l}\text { h Calochortus barbatus (Kunth) } \\
\text { J.H. Painter }\end{array}$ & $12128(2)$ & \\
\hline \multicolumn{3}{|l|}{ NOLINACEAE } \\
\hline ar Calibanus hookeri (Lem.) Trel. (A) & JB & $\begin{array}{c}\text { Zona Árida Queretano } \\
\text { Hidalguense }\end{array}$ \\
\hline $\begin{array}{l}\text { ar Dasylirion acrotrichum (Schldtl.) } \\
\text { Zucc. (A) }\end{array}$ & JB & \\
\hline ar Dasylirion longissimum Lem. (A) & JB & \\
\hline \multicolumn{3}{|l|}{ ORCHIDACEAE } \\
\hline h Mesadenus polyanthus (Rchb. f.) Schltr. & $12126(2)$ & \\
\hline \multicolumn{3}{|l|}{ POACEAE } \\
\hline h Stipa ichu (Ruiz \& Pav.) Kunth & $12133(3)$ & \\
\hline \multicolumn{3}{|l|}{ ACANTHACEAE } \\
\hline ar Anisacanthus quadrifidus (Vahl) Nees & $12153(2)$ & \\
\hline $\begin{array}{l}\text { ar Carlowrightia parviflora (Buckl.) } \\
\text { Wassh. }\end{array}$ & $\begin{array}{c}10956,11319 \\
12094(1,2)\end{array}$ & \\
\hline \multicolumn{3}{|l|}{ ANACARDIACEAE } \\
\hline Ar Bonetiella anomala (I.M. Johnst.) Rzed. & $12176,12174(2)$ & Desierto Chihuahuense \\
\hline
\end{tabular}


Apéndice. Continuación.

\begin{tabular}{|c|c|c|}
\hline TAXON & Núm. de colecta & Amplitud geográfica \\
\hline $\begin{array}{l}\text { ar Rhus microphylla } \\
\text { Engelm. ex A. Gray }\end{array}$ & $\begin{array}{c}12102,12109 \\
(1,2,3)\end{array}$ & $\begin{array}{l}\text { Zonas áridas del Norte de } \\
\text { México }\end{array}$ \\
\hline \multicolumn{3}{|l|}{ APIACEAE } \\
\hline ar Arracacia tolucensis (Kunth) Hemsl. & $12069(1,2,3)$ & \\
\hline \multicolumn{3}{|l|}{ APOCYNACEAE } \\
\hline ar Mandevilla foliosa (Müll. Arg.) Hemsl. & $12047(1,2,3)$ & \\
\hline ar Mandevilla syrinx Woodson & $12039(1,2,3)$ & $\begin{array}{c}\text { Vertiente pacífica y centro } \\
\text { de México }\end{array}$ \\
\hline \multicolumn{3}{|l|}{ ASCLEPIADACEAE } \\
\hline $\mathrm{h}$ Asclepias mexicana Cav. & $12116(1,2,3)$ & \\
\hline ar Matelea chrysantha (Greenm.) Woodson & $12050(2,3)$ & \\
\hline \multicolumn{3}{|l|}{ ASTERACEAE (COMPOSITAE) } \\
\hline $\begin{array}{l}\text { h Acourtia purpusii (Brandg.) } \\
\text { Reveal \& R.M. King. }\end{array}$ & $12156(2)$ & \\
\hline $\mathrm{h}$ Ambrosia psilostachya $\mathrm{DC}$. & $12096(1,2,3)$ & \\
\hline ar Baccharis pteronioides DC. & $\begin{array}{c}12129,11976 \\
12015,12021 \\
(1,2,3)\end{array}$ & \\
\hline $\begin{array}{l}\text { h Bahia absinthifolia subsp. absinthifolia } \\
\text { Benth. }\end{array}$ & $12155(1,3)$ & \\
\hline h Chaptalia nutans (L.) Polak. & $12078(2)$ & \\
\hline h Chrysactinia mexicana A. Gray & $\begin{array}{c}12139,12135 \\
(1,2)\end{array}$ & \\
\hline ar Coreopsis mutica DC. & $12005(3)$ & \\
\hline h Dahlia coccinea Cav. & $12070(1,2,3)$ & \\
\hline $\begin{array}{l}\text { h Dyscritothamnus mirandae } \\
\text { Paray }\end{array}$ & $12171(2)$ & $\begin{array}{l}\text { Zona Árida Queretano } \\
\text { Hidalguense } \\
\end{array}$ \\
\hline $\mathrm{h}$ Eupatorium deltoideum Jacq. & $12184 b(2)$ & \\
\hline $\mathrm{h}$ Eupatorium espinosarum A. Gray & $12137(3)$ & \\
\hline $\begin{array}{l}\text { ar Flourensia resinosa (Brandegee) } \\
\text { S.F. Blake }\end{array}$ & JB & $\begin{array}{l}\text { Zona Árida Queretano } \\
\text { Hidalguense } \\
\end{array}$ \\
\hline Ar Gochnatia hypolueuca (DC.) A. Gray & $12170(2)$ & \\
\hline h Gymnosperma glutinosum (Spreng.) Less. & $12173(2)$ & \\
\hline ar Montanoa tomentosa Cerv. & $12134(2,3)$ & \\
\hline
\end{tabular}


Apéndice. Continuación.

\begin{tabular}{|c|c|c|}
\hline TAXON & Núm. de colecta & Amplitud geográfica \\
\hline ar Parthenium incanum Kunth & $\begin{array}{c}12057,12088 \\
(1,2,3)\end{array}$ & Desierto Chihuahuense \\
\hline ar Perymenium mendezii DC. & $\begin{array}{l}10764,12048 \\
12056(1,2,3)\end{array}$ & \\
\hline ar Pinaropappus roseus (Less.) Less. & $12195(2)$ & \\
\hline $\begin{array}{l}\text { ar Pittocaulon praecox (Cav.) } \\
\text { H. Rob. \& Brettell }\end{array}$ & JB & \\
\hline h Sanvitalia ocymoides DC. & $12110(1,2,3)$ & \\
\hline h Sanvitalia procumbens Lam. & JB & \\
\hline ar Senecio albo-lutescens Sch. Bip. & $12060(3)$ & \\
\hline ar Stevia salicifolia Cav. & $12161(2)$ & \\
\hline ar Stevia tomentosa Kunth & $12142(2)$ & \\
\hline $\begin{array}{c}\text { h Verbesina encelioides (Cav.) } \\
\text { Benth. \& Hook. ex A. Gray }\end{array}$ & $12097(1,2,3)$ & \\
\hline h Verbesina oreopola Rob. \& Greenm. & $12118(1,2,3)$ & \\
\hline \multicolumn{3}{|l|}{ BERBERIDACEAE } \\
\hline ar Berberis albicans Zamudio \& Marroquín & $12112(1,2,3)$ & \\
\hline \multicolumn{3}{|l|}{ BORAGINACEAE } \\
\hline $\begin{array}{l}\text { h Tiquilia purpusii (Brandegee) A.T. } \\
\text { Richardson }\end{array}$ & $12080(2,3)$ & \\
\hline \multicolumn{3}{|l|}{ CACTACEAE } \\
\hline $\begin{array}{l}\text { Gt Ariocarpus kotschoubeyanus (Lem.) } \\
\text { K. Schum. (Pr) }\end{array}$ & $080,177(1,3) \mathrm{JB}$ & Desierto Chihuahuense \\
\hline $\begin{array}{l}\text { g Astrophytum ornatum (DC.) } \\
\text { Britton \& Rose }(\mathbf{A})\end{array}$ & $189(1,4) \mathrm{JB}$ & $\begin{array}{c}\text { Zona Árida Queretano } \\
\text { Hidalguense }\end{array}$ \\
\hline Cc Coryphantha erecta (Lem.) Lem. & $202,224(3,4) \mathrm{JB}$ & Desierto Chihuahuense \\
\hline $\begin{array}{l}\text { c Coryphantha octacantha (DC.) } \\
\text { Britton \& Rose }\end{array}$ & $\begin{array}{c}219,156(1,2,3 \\
4) \mathrm{JB}\end{array}$ & Desierto Chihuahuense \\
\hline $\begin{array}{l}\text { g Coryphantha radians (DC.) } \\
\text { Britton \& Rose }\end{array}$ & $104,205(3,4) \mathrm{JB}$ & \\
\hline $\begin{array}{l}\text { ar Cylindropuntia imbricata (Haw.) } \\
\text { F.M. Knuth }\end{array}$ & $\begin{array}{l}158,168,169 \\
207,217,222 \\
(1,2,3,4) \mathrm{JB}\end{array}$ & Desierto Chihuahuense \\
\hline $\begin{array}{l}\text { ar Cylindropuntia kleiniae (DC.) } \\
\text { F.M. Knuth }\end{array}$ & $\begin{array}{c}154,160 \\
(1,2,3,4) \mathrm{JB}\end{array}$ & \\
\hline
\end{tabular}


Apéndice. Continuación.

\begin{tabular}{|c|c|c|}
\hline TAXON & Núm. de colecta & Amplitud geográfica \\
\hline $\begin{array}{l}\text { ar Cylindropuntia leptocaulis (DC.) } \\
\text { F.M. Knuth }\end{array}$ & $\begin{array}{c}161,206 \\
(1,2,3) \mathrm{JB}\end{array}$ & \\
\hline g Echinocactus grusonii Hildm. $(\mathbf{P})$ & $\mathrm{JB}$ & Semidesierto Queretano \\
\hline $\begin{array}{l}\text { g Echinocactus platyacanthus } \\
\text { Link \& Otto (Pr) }\end{array}$ & $\begin{array}{c}154,160 \\
(1,2,3,4) \mathrm{JB}\end{array}$ & \\
\hline $\begin{array}{l}\text { Cc Echinocereus cinerascens (DC.) } \\
\text { Rumpler }\end{array}$ & $\begin{array}{c}161,206 \\
(1,2,3,4) \mathrm{JB}\end{array}$ & \\
\hline Cc Echinocereus pentalophus (DC.) Lem. & $128(3,4) \mathrm{JB}$ & \\
\hline $\begin{array}{l}\text { Cc Echinocereus schmollii (Weing.) } \\
\text { N.P. Taylor (P) }\end{array}$ & $125,126(3,4) \mathrm{JB}$ & Semidesierto Queretano \\
\hline $\begin{array}{l}\text { g Ferocactus echidne (DC.) } \\
\text { Br. \& R. }\end{array}$ & $\begin{array}{c}128,134 \\
(1,2,3,4) \mathrm{JB}\end{array}$ & Desierto Chihuahuense \\
\hline $\begin{array}{l}\text { g Ferocactus glaucescens (DC.) } \\
\text { Br. \& R. }\end{array}$ & $148(1,2,3,4) \mathrm{JB}$ & Desierto Chihuahuense \\
\hline $\begin{array}{l}\text { g Ferocactus histrix (DC.) } \\
\text { G. E. Linds (Pr) }\end{array}$ & $143,144(1,4) \mathrm{JB}$ & \\
\hline $\begin{array}{l}\text { g Ferocactus latispinus (Haw.) } \\
\text { Britton \& Rose }\end{array}$ & $218(3,4) \mathrm{JB}$ & \\
\hline $\begin{array}{l}\text { Ab Isolatocereus dumortieri (Scheidw.) } \\
\text { Backeb. }\end{array}$ & $\begin{array}{c}115,197,250 \\
(2,3,4) \mathrm{JB}\end{array}$ & \\
\hline $\begin{array}{l}\text { g Lophophora diffusa (Croizat) } \\
\text { Bravo (A) }\end{array}$ & $\begin{array}{c}182,200 \\
(1,3,4) \mathrm{JB}\end{array}$ & Semidesierto Queretano \\
\hline $\begin{array}{l}\text { Gc Mammillaria compressa subsp. compressa } \\
\text { (Schum.) Borg. }\end{array}$ & $198(3,4) \mathrm{JB}$ & Desierto Chihuahuense \\
\hline g Mammillaria crinita DC subsp. crinita & $\begin{array}{l}076,092,122 \\
(3,4) \mathrm{JB}\end{array}$ & Desierto Chihuahuense \\
\hline $\begin{array}{l}\text { g Mammillaria crinita subsp. painteri } \\
\text { (Rose ex Quehl) U. Guzmán (Pr) }\end{array}$ & $192(3,4) \mathrm{JB}$ & $\begin{array}{l}\text { Zona Árida Queretano } \\
\text { Hidalguense }\end{array}$ \\
\hline $\begin{array}{l}\text { g Mammillaria decipiens subsp. } \\
\text { camptotricha (Dams) D. R. Hunt }\end{array}$ & $\begin{array}{l}091,199,124 \\
(1,2,3,4) \mathrm{JB}\end{array}$ & Desierto Chihuahuense \\
\hline Cc Mammillaria elongata DC. & $\begin{array}{c}103,153,211, \\
234,235,236 \\
(1,2,3) \mathrm{JB}\end{array}$ & Desierto Chihuahuense \\
\hline Gc Mammillaria gracilis Pfeiff. & $097(3,4) \mathrm{JB}$ & $\begin{array}{l}\text { Zona Árida Queretano } \\
\text { Hidalguense }\end{array}$ \\
\hline g Mammillaria herrerae Werderm. (P) & JB & Semidesierto Queretano \\
\hline
\end{tabular}


Apéndice. Continuación.

\begin{tabular}{|c|c|c|}
\hline TAXON & Núm. de colecta & Amplitud geográfica \\
\hline g Mammillaria longimamma DC (A) & $\begin{array}{c}075,127,196 \\
(3,4) \mathrm{JB}\end{array}$ & $\begin{array}{c}\text { Zona Árida Queretano } \\
\text { Hidalguense }\end{array}$ \\
\hline g Mammillaria magnimamma Haw. & $083(3)$ & \\
\hline Cc Mammillaria microhelia Werderm. (Pr) & $073(1,3,4) \mathrm{JB}$ & $\begin{array}{l}\text { Zona Árida Queretano } \\
\text { Hidalguense }\end{array}$ \\
\hline g Mammillaria muehlenpfordtii C.F. Föerster & $\begin{array}{c}166,167 \\
(1,3,4) \mathrm{JB}\end{array}$ & Desierto Chihuahuense \\
\hline Gc Mammillaria parkinsonii C. Ehrenb. (Pr) & $107,190(3,4) \mathrm{JB}$ & $\begin{array}{c}\text { Zona Árida Queretano } \\
\text { Hidalguense }\end{array}$ \\
\hline $\begin{array}{l}\text { g Mammillaria perbella } \\
\text { Hildm. ex K. Schum. }\end{array}$ & $210(3,4) \mathrm{JB}$ & $\begin{array}{c}\text { Zona Árida Queretano } \\
\text { Hidalguense }\end{array}$ \\
\hline $\begin{array}{l}\text { g Mammillaria polythele subsp. durispina } \\
\text { (Boed.) D.R. Hunt }\end{array}$ & $\begin{array}{l}090,108,171 \\
(1,2,3,4) \mathrm{JB}\end{array}$ & $\begin{array}{c}\text { Zona Árida Queretano } \\
\text { Hidalguense }\end{array}$ \\
\hline $\begin{array}{l}\text { g Mammillaria pseudocrucigera } \\
\text { R.T. Craig }\end{array}$ & $178,102(3) \mathrm{JB}$ & \\
\hline $\begin{array}{l}\text { g Mammillaria schiedeana Ehrenb. subsp. } \\
\text { dumetorum (J.A. Purpus) D.R. Hunt (Pr) }\end{array}$ & $214,215(1,3) \mathrm{JB}$ & $\begin{array}{c}\text { Zona Árida Queretano } \\
\text { Hidalguense }\end{array}$ \\
\hline g Mammillaria uncinata Zucc. ex Pfeiff. & $086(1,2,3,4) \mathrm{JB}$ & \\
\hline $\begin{array}{l}\mathrm{Ab} \text { Marginatocereus marginatus (DC.) } \\
\text { Backeb. }\end{array}$ & $142(3,4) \mathrm{JB}$ & \\
\hline $\begin{array}{l}\text { Ab Myrtillocactus geometrizans (Martius) } \\
\text { Console }\end{array}$ & $\begin{array}{l}139,223,249 \\
(1,2,3,4) \mathrm{JB}\end{array}$ & Desierto Chihuahuense \\
\hline $\begin{array}{l}\text { c Neolloydia conoidea (DC.) } \\
\text { Britton \& Rose }\end{array}$ & $\begin{array}{c}077,195 \\
(1,3,4) \mathrm{JB}\end{array}$ & Desierto Chihuahuense \\
\hline ar Opuntia cantabrigiensis Lynch. & $\begin{array}{l}193,225 \\
(1,3,4) \mathrm{JB}\end{array}$ & \\
\hline Ab Opuntia leucotricha DC. & $164(2,3,4) \mathrm{JB}$ & Desierto Chihuahuense \\
\hline ar Opuntia microdasys (Lehm.) Pfeiff. & $\begin{array}{l}151,201,208 \\
(1,2,3,4) \mathrm{JB}\end{array}$ & Desierto Chihuahuense \\
\hline ar Opuntia pubescens H. L. Wendl. ex Pfeiff. & $204,240(3)$ & \\
\hline ar Opuntia robusta H. L. Wendl. ex Pfeiff. & $\begin{array}{c}138,221,253 \\
(1,2,3) \mathrm{JB}\end{array}$ & \\
\hline ar Opuntia stenopetala Engelm. & $\begin{array}{l}152,172,191 \\
(1,2,3,4) \mathrm{JB}\end{array}$ & Desierto Chihuahuense \\
\hline Ab Opuntia streptacantha Lem. & $175,233(1,3) \mathrm{JB}$ & \\
\hline
\end{tabular}


Apéndice. Continuación.

\begin{tabular}{|c|c|c|}
\hline TAXON & Núm. de colecta & Amplitud geográfica \\
\hline Ab Opuntia tomentosa Salm-Dyck & $\begin{array}{l}145,146,256 \\
257(1,3,4) \mathrm{JB}\end{array}$ & \\
\hline $\begin{array}{l}\text { g Stenocactus crispatus (DC.) } \\
\text { Berger ex Hill }\end{array}$ & JB & \\
\hline $\begin{array}{l}\text { g Stenocactus obvallatus (DC.) } \\
\text { Berger ex Hill }\end{array}$ & $\begin{array}{c}110,111 \\
(1,3,4) \mathrm{JB}\end{array}$ & \\
\hline $\begin{array}{l}\text { g Stenocactus sulphureus (A. Dietri.) } \\
\text { Bravo (Pr) }\end{array}$ & JB & Semidesierto Queretano \\
\hline Ab Stenocereus griseus (Haw.) Buxb. & $209(1,3,4) \mathrm{JB}$ & \\
\hline $\begin{array}{l}\text { g Strombocactus disciformis (DC.) } \\
\text { Britton \& Rose (A) }\end{array}$ & $105,106(3,4) \mathrm{JB}$ & $\begin{array}{l}\text { Zona Árida Queretano } \\
\text { Hidalguense }\end{array}$ \\
\hline $\begin{array}{l}\text { c Thelocactus hastifer (Werderm. \& Boed.) } \\
\text { F.M. Knuth (Pr) }\end{array}$ & $\begin{array}{l}132,133,181 \\
194(2,3,4) \mathrm{JB}\end{array}$ & Semidesierto Queretano \\
\hline $\begin{array}{l}\text { c Thelocactus leucacanthus } \\
\text { (Zucc. ex Pfeiff.) Britton \& Rose } \\
\text { subsp. leucacanthus }\end{array}$ & $\begin{array}{l}082,140,157 \\
(2,3,4) \mathrm{JB}\end{array}$ & Desierto Chihuahuense \\
\hline $\begin{array}{l}\text { g Turbinicarpus pseudomacrochele subsp. } \\
\text { pseudomacrochele (Backeb.) Buxb. \& } \\
\text { Backeb. (P) }\end{array}$ & $\begin{array}{c}179,184,185 \\
186(1,2,3,4) \mathrm{JB}\end{array}$ & $\begin{array}{l}\text { Zona Árida Queretano } \\
\text { Hidalguense }\end{array}$ \\
\hline \multicolumn{3}{|l|}{ CAPRIFOLIACEAE } \\
\hline ar Lonicera pilosa (Kunth) Willd. & $12166(2)$ & Norte y Centro de Méxicc \\
\hline $\begin{array}{l}\text { ar Symphoriocarpos microphyllus } \\
\text { Kunth }\end{array}$ & $12162(2)$ & \\
\hline \multicolumn{3}{|l|}{ CELASTRACEAE } \\
\hline ar Mortonia greggii Gray & $12215(2)$ & \\
\hline \multicolumn{3}{|l|}{ CISTACEAE } \\
\hline h Helianthemum patens Hemsl. & 12224 & Altiplano Mexicano \\
\hline \multicolumn{3}{|l|}{ CONVULVULACEAE } \\
\hline h Evolvulus prostratus Rob. & $12044(1,2,3)$ & \\
\hline h Ipomoea ciervensis Painter & $12059(1,2,3)$ & $\begin{array}{l}\text { Zona Árida Queretano } \\
\text { Hidalguense }\end{array}$ \\
\hline e Ipomoea costellata Torr. & $\begin{array}{c}11327,11532 \\
11959,12106 \\
(1,2,3)\end{array}$ & \\
\hline
\end{tabular}


Apéndice. Continuación.

\begin{tabular}{|c|c|c|}
\hline TAXON & Núm. de colecta & Amplitud geográfica \\
\hline Ar Ipomoea murucoides Roem. \& Schult. & JB & \\
\hline $\begin{array}{l}\text { Ar Ipomoea rzedowskii E. Carranza, S. } \\
\text { Zamudio \& G. Munguía }\end{array}$ & $12175(2)$ & $\begin{array}{c}\text { Zona Árida Queretano } \\
\text { Hidalguense }\end{array}$ \\
\hline \multicolumn{3}{|l|}{ CORNACEAE } \\
\hline Ar Cornus disciflora DC. & $12201(2)$ & \\
\hline \multicolumn{3}{|l|}{ CRASSULACEAE } \\
\hline h Echeveria rosea Lindley & $\begin{array}{c}12223 \\
(1,2,3,4) \mathrm{JB}\end{array}$ & \\
\hline $\mathrm{h}$ Pachyphytum viride Walther & $180(3,4)(\mathrm{JB})$ & $\begin{array}{c}\text { Zona Árida Queretano } \\
\text { Hidalguense }\end{array}$ \\
\hline h Sedum greggii Hemsl. & $\begin{array}{c}12188 \\
(1,2,3,4) \mathrm{JB}\end{array}$ & \\
\hline \multicolumn{3}{|l|}{ ERICACEAE } \\
\hline Ar Arbutus xalapensis Kunth & $12208(2)$ & \\
\hline ar Arctostaphylos discolor (Hook.) DC. & $12157(2)$ & \\
\hline \multicolumn{3}{|l|}{ EUPHORBIACEAE } \\
\hline ar Croton morifolius Willd. & $\begin{array}{l}12107,12090 \\
12007(1,2,3)\end{array}$ & \\
\hline h Euphorbia antisyphilitica Zucc. & JB & \\
\hline h Euphorbia furcillata Kunth & $12184 \mathrm{aa}(2)$ & \\
\hline ar Stillingia sanguinolenta Muell. Arg. & $12031(1,2,3)$ & \\
\hline \multicolumn{3}{|l|}{ FABACEAE } \\
\hline Ar Acacia berlandieri Benth. & $12211(2)$ & \\
\hline $\begin{array}{l}\text { ar Acacia farnesiana (L.) Willd. } \\
\text { subsp.farnesiana }\end{array}$ & $12205(2,4)$ & \\
\hline Ar Acacia reniformis Benth. & $12152(2,4)$ & $\begin{array}{c}\text { Zona Árida Queretano } \\
\text { Hidalguense } \\
\end{array}$ \\
\hline Ar Acacia roemeriana Scheele & $12178,12183(2)$ & \\
\hline ar Astragalus hypoleucus Schauer & $12079(3)$ & \\
\hline Ar Bauhinia ramosissima Benth. & $12113(1,2,3)$ & \\
\hline ar Calliandra grandiflora (L' Hér.) Benth. & $12051(2)$ & \\
\hline $\begin{array}{l}\text { h Crotalaria rotundifolia (Walt.) Gmelin } \\
\text { var. vulgaris Windler }\end{array}$ & $12199(2)$ & \\
\hline ar Dalea zimapanica Schauer & $12124(1,2,3)$ & \\
\hline
\end{tabular}


Apéndice. Continuación.

\begin{tabular}{|c|c|c|}
\hline TAXON & Núm. de colecta & Amplitud geográfica \\
\hline ar Desmodium orbiculare Schldl. & $12145(2,3)$ & \\
\hline Ar Diphysa suberosa S. Watson & $\begin{array}{c}12089,12100 \\
(1,2,3)\end{array}$ & \\
\hline Ar Eysenhardtia polystachya (Ort.) Sarg. & $12077(1,2,3)$ & \\
\hline Ar Harpalyce arborescens A. Gray & $12222,12121(2)$ & \\
\hline ar Hoffmanseggia arida Ros. & $12052(1,2,3)$ & \\
\hline Ar Indigofera suffruticosa Mill. & $\begin{array}{c}12092,12184 \\
(1,2,3)\end{array}$ & \\
\hline Ar Leucaena cuspidata Standley & $12229,12158(2)$ & \\
\hline h Lupinus aff. elegans H.B.K. & $12194(2)$ & \\
\hline h Lupinus aff. exaltatus Zucc. & $11139(2)$ & \\
\hline Ar Lysiloma microphyllum Benth. & $12151(2)$ & \\
\hline ar Mimosa aculeaticarpa Ortega & $\begin{array}{c}12067,12120 \\
(1,2,3)\end{array}$ & \\
\hline ar Mimosa leucaenoides Benth. & $\begin{array}{c}12221,12219, \\
12210(2)\end{array}$ & \\
\hline h Phaseolus acutifolius A. Gray & $\begin{array}{c}12038,12111 \\
(1,2,3)\end{array}$ & \\
\hline h Phaseolus atropurpurens DC. & $12098(1,2)$ & \\
\hline ar Pithecellobium revolutum Rose. & $12085(1,2,3)$ & \\
\hline Ar Sophora secundiflora Lag. ex DC. & $12225(2)$ & \\
\hline ar Tephrosia leucantha Kunth & $12033(1,2,3)$ & \\
\hline h Vicia humilis Kunth & $12163(2)$ & \\
\hline \multicolumn{3}{|l|}{ FAGACEAE } \\
\hline Ar Quercus crassifolia Humb. \& Bonpl. & $10846(2)$ & \\
\hline Ar Quercus dysophylla Benth. & $12061(1,2,3)$ & \\
\hline Ar Quercus glaucoides Mart. \& Gal. & $10725(2,3)$ & \\
\hline Ar Quercus greggii (A. DC.) Trel. & $12167(2)$ & \\
\hline Ar Quercus grisea Liebm. & $12054(2,3)$ & \\
\hline Ar Quercus mexicana Humb. \& Bonpl. & $12168,12160(2)$ & \\
\hline Ar Quercus obtusata Humb. \& Bonpl. & $10849(2)$ & \\
\hline Ar Quercus polymorpha Schltdl. \& Cham. & $12217,12196(2)$ & \\
\hline Ar Quercus pulchella Cham. \& Schldl. & $12055(1,2,3)$ & \\
\hline
\end{tabular}


Apéndice. Continuación.

\begin{tabular}{|c|c|c|}
\hline TAXON & Núm. de colecta & Amplitud geográfica \\
\hline \multicolumn{3}{|l|}{ FLACOURTIACEAE } \\
\hline $\begin{array}{l}\text { ar Neopringlea integrifolia (Hemsl.) } \\
\text { S. Watson }\end{array}$ & $\begin{array}{c}2114,12146 \\
(1,2,3)\end{array}$ & $\begin{array}{c}\text { Parte alta de la cuenca del } \\
\text { Pánuco }\end{array}$ \\
\hline \multicolumn{3}{|l|}{ FOUQUIERIACEAE } \\
\hline $\begin{array}{l}\text { ar Fouquieria splendens Engelm. } \\
\text { subsp. breviflora Henr. }\end{array}$ & JB & Zonas áridas del Altiplano \\
\hline \multicolumn{3}{|l|}{ GARRYACEAE } \\
\hline ar Garrya ovata Benth. & $12162(2)$ & Desierto Chihuahuense \\
\hline \multicolumn{3}{|l|}{ GENTIANACEAE } \\
\hline $\mathrm{h}$ Centaurium quitense (Kunth) Rob. & $\begin{array}{c}12006,12191 \\
(2,3)\end{array}$ & \\
\hline h Geniostemon coulteri Engelm. \& A. Gray & $12212(2)$ & $\begin{array}{l}\text { Zona Árida Queretano } \\
\text { Hidalguense }\end{array}$ \\
\hline h Gentiana spathacea Kunth (Pr) & $12190,12165(2)$ & $\begin{array}{l}\text { Sierra Madre Oriental y } \\
\text { Sierra Madre Occidental }\end{array}$ \\
\hline \multicolumn{3}{|l|}{ KRAMERIACEAE } \\
\hline ar Krameria cytisoides Cav. & $12068(1,2,3)$ & Desierto Chihuahuense \\
\hline \multicolumn{3}{|l|}{ LAMIACEAE (LABIATAE) } \\
\hline ar Hyptis albida Kunth & $12218(2)$ & \\
\hline ar Poliomintha marifolia Benth. & $\begin{array}{l}12172,12220 \\
12075(2)\end{array}$ & \\
\hline h Salvia candicans Mart. \& Gal. & $\begin{array}{c}12154,12119 \\
(1,2,3)\end{array}$ & \\
\hline h Salvia connivens Epling & $121408(2,3)$ & \\
\hline ar Salvia keerlii Benth. & $\begin{array}{c}12141,12138 \\
(2,3)\end{array}$ & \\
\hline h Salvia regla $\mathrm{Cav}$. & $12132(1,2,3)$ & \\
\hline h Salvia riparia Kunth & $12180(2)$ & \\
\hline h Scutellaria caerulea Moc. \& Sessé ex Benth. & $12073(2,3)$ & \\
\hline h Teucrium cubense Jacq.. & $12207(2)$ & \\
\hline \multicolumn{3}{|l|}{ LAURACEAE } \\
\hline Ar Litsea glaucescens Kunth (P) & $12164(2)$ & \\
\hline \multicolumn{3}{|l|}{ LOASACEAE } \\
\hline h Cevallia sinuata Lag. & $12125(1,2,3)$ & Desierto Chihuahuense \\
\hline
\end{tabular}


Apéndice. Continuación.

\begin{tabular}{|c|c|c|}
\hline TAXON & Núm. de colecta & Amplitud geográfica \\
\hline \multicolumn{3}{|l|}{ LORANTACEAE } \\
\hline e Phoradendron bolleanum (Seem.) Eichler & $12108(1,2,3)$ & \\
\hline e Psittacanthus calyculatus (DC.) G. Don & $12184 \mathrm{a}(2)$ & \\
\hline \multicolumn{3}{|l|}{ MALPIGHIACEAE } \\
\hline ar Galphimia glauca Cav. & $12091(1,3)$ & \\
\hline \multicolumn{3}{|l|}{ MALVACEAE } \\
\hline h Malvastrum americanum (L.) Torrey & $12099(1,2,3)$ & \\
\hline \multicolumn{3}{|l|}{ OLEACEAE } \\
\hline ar Forestiera phillyreoides (Benth.) Torrey & $12062(1,2,3)$ & \\
\hline $\begin{array}{l}\text { h Menodora helianthemoides } \\
\text { Humb. \& Bonpl. }\end{array}$ & $12104(1,2)$ & \\
\hline $\mathrm{h}$ Menodora coulteri A. Gray & $10615(2)$ & \\
\hline \multicolumn{3}{|l|}{ PASSIFLORACEAE } \\
\hline h Passiflora bryoniodes Kunth & $12049(2,3)$ & \\
\hline h Passiflora subpeltata Ort. & $12189(2)$ & \\
\hline \multicolumn{3}{|l|}{ PHYTOLACACEAE } \\
\hline ar Phytolacca icosandra $\mathrm{L}$. & $12058(1,2,3)$ & \\
\hline \multicolumn{3}{|l|}{ PIPERACEAE } \\
\hline h Peperomia cumpylotropa Hill. & $\begin{array}{l}10308,11817 \\
(1,2,3,4) \mathrm{JB} \\
\end{array}$ & \\
\hline \multicolumn{3}{|l|}{ POLYGALACEAE } \\
\hline h Polygala compacta Rose & $\begin{array}{l}12213,12034 \\
12123(1,2,3)\end{array}$ & \\
\hline \multicolumn{3}{|l|}{ PRIMULACEAE } \\
\hline h Samolus ebracteatus Kunth & $12169(2)$ & \\
\hline \multicolumn{3}{|l|}{ PTEROSTEMONACEAE } \\
\hline ar Pterostemon mexicanus Schauer & $12122(1,3)$ & $\begin{array}{c}\text { Zona Árida Queretano } \\
\text { Hidalguense } \\
\end{array}$ \\
\hline \multicolumn{3}{|l|}{ RAFFLESIACEAE } \\
\hline h Pilostyles thurberi A. Gray & $\begin{array}{l}12043,12028 \\
12063(1,2,3) \\
\end{array}$ & \\
\hline \multicolumn{3}{|l|}{ RHAMNACEAE } \\
\hline ar Ceanothus caeruleus Lag. & $12204(2)$ & \\
\hline
\end{tabular}


Apéndice. Continuación.

\begin{tabular}{|c|c|c|}
\hline TAXON & Núm. de colecta & Amplitud geográfica \\
\hline ar Karwinskia humboldtiana Roem. \& Schult. & $12147(2)$ & \\
\hline \multicolumn{3}{|l|}{ ROSACEAE } \\
\hline Ar Cercocarpus macrophyllus Schneid. & $12200,12185(2)$ & Endémica de México \\
\hline \multicolumn{3}{|l|}{ RUBIACEAE } \\
\hline ar Cigarrilla mexicana (Zucc. \& Mart.) Aiello & $\begin{array}{c}12227,12149 \\
12181(2) \\
\end{array}$ & $\begin{array}{c}\text { Zona Árida Queretano } \\
\text { Hidalguense }\end{array}$ \\
\hline h Houstonia rubra Cav. & $12074(2,3)$ & \\
\hline Ar Randia capitata Sessé \& Moc. ex DC. & $12084(2,3)$ & \\
\hline \multicolumn{3}{|l|}{ SAPINDACEAE } \\
\hline ar Cardiospermum halicacabum L. & $12046(1,2,3)$ & \\
\hline \multicolumn{3}{|l|}{ SCROPHULARIACEAE } \\
\hline h Hemichaena coulteri A. Gray & $12214(2)$ & \\
\hline h Lamouroxia dasyantha (Cham. \& Schl.) & $12131(1,2,3)$ & \\
\hline h Mimulus glabratus Kunth & $12187(2)$ & \\
\hline h Russelia polyedra Zucc. & $12072(1,2,3)$ & \\
\hline \multicolumn{3}{|l|}{ SOLANACEAE } \\
\hline $\begin{array}{l}\text { ar Capsicum annuum var. aviculare (Dierb.) } \\
\text { D'Arcy \& Eshbaugh }\end{array}$ & $\begin{array}{l}11558,12130 \\
12093(1,2,3)\end{array}$ & \\
\hline h Leptoglossis coulteri A. Gray & $\begin{array}{c}12076,12105 \\
(1,2,3)\end{array}$ & \\
\hline h Solanum tridynamum Dunal. & $\begin{array}{c}9957,11939 \\
(1,2,3)\end{array}$ & \\
\hline \multicolumn{3}{|l|}{ STERCULACEAE } \\
\hline ar Ayenia rotundifolia Hemsl. & $12101(1,2)$ & $\begin{array}{c}\text { Zona Árida Queretano } \\
\text { Hidalguense }\end{array}$ \\
\hline \multicolumn{3}{|l|}{ THEACEAE } \\
\hline Ar Ternstroemia sylvatica Schdl. \& Cham. & $12202(2)$ & Sierra Madre Oriental \\
\hline \multicolumn{3}{|l|}{ TURNERACEAE } \\
\hline h Turnera diffusa Willd. ex Schult. & $12103(2)$ & \\
\hline \multicolumn{3}{|l|}{ ULMACEAE } \\
\hline Ar Celtis caudata Planch. & $\begin{array}{c}12179,12027 \\
(1,2,3)\end{array}$ & \\
\hline Ar Celtis aff. iguanaea (Jacq.) Sarg. & $\begin{array}{c}11340,12029 \\
(1,2,3)\end{array}$ & \\
\hline
\end{tabular}


Apéndice. Continuación.

\begin{tabular}{|c|c|c|}
\hline TAXON & Núm. de colecta & Amplitud geográfica \\
\hline VALERIANACEAE & & \\
\hline h Valeriana subincisa Benth. & $12203(2)$ & \\
\hline VERBENACEAE & & \\
\hline ar Citharexylum altamiranum Greenm. & $12144(2,3)$ & Altiplano mexicano \\
\hline ar Citharexylum lycioides D. Don & $12026(1,2,3)$ & Altiplano mexicano \\
\hline ar Citharexylum oleinum Moldenke & 12159,12136, & Sierra Madre Oriental \\
& $12032(1,2,3)$ & \\
\hline ar Lantana camara L. & $12045(1,2,3)$ & \\
\hline
\end{tabular}

